\title{
Multiple roles of the transcription factor AtMYBR1/AtMYB44 in ABA signaling, stress responses, and leaf senescence
}

\author{
Masrur R Jaradat ${ }^{1}$, J Allan Feurtado ${ }^{1}$, Daiqing Huang ${ }^{1}$, Yongquan Lu $^{2}$ and Adrian J Cutler ${ }^{1 *}$
}

\begin{abstract}
Background: The transcription factor AtMYBR1 (MYB44) is a member of the MYB family of transcription factors and is expressed throughout the plant life cycle and especially in senescing and wounded leaves. It has previously been shown to be involved in responses to abiotic stress and is regulated by phosphorylation.

Results: When MYBR1 was over-expressed under the control of the constitutive 355 promoter in Arabidopsis thaliana (OxMYBR1), leaf senescence was delayed. In contrast loss-of-function mybr1 plants showed more rapid chlorophyll loss and senescence. The MYBR1 promoter strongly drove $\beta$-GLUCURONIDASE reporter gene expression in tissues immediately after wounding and many wounding/pathogenesis genes were downregulated in OXMYBR1. OXMYBR1 plants were more susceptible to injury under water stress than wildtype, which was correlated with suppression of many ABA inducible stress genes in OxMYBR1. Conversely, mybr1 plants were more tolerant of water stress and exhibited reduced rates of water loss from leaves. MYBR1 physically interacted with ABA receptor PYR1-LIKE8 (PYL8) suggesting a direct involvement of MYBR1 in early ABA signaling. MYBR1 appears to exhibit partially redundant functions with AtMYBR2 (MYB77) and double mybr1 X mybr2 mutants exhibited stronger senescence and stress related phenotypes than single mybr1 and mybr2 mutants.
\end{abstract}

Conclusions: MYBR1 is a negative regulator of ABA, stress, wounding responses and blocks senescence. It appears to have a homeostatic function to maintain growth processes in the event of physical damage or stress.

Keywords: ABA, Drought stress, Transcription factor, PYL8, Senescence

\section{Background}

In order to acclimate and protect themselves, plants translate environmental challenges such as drought, waterlogging, extreme temperatures, soil salinity, wounding, and pathogen attack into internal signals through hormones, second messengers, and transcription factors (TFs). The phytohormone abscisic acid (ABA) regulates abiotic stress responses and other vital processes in plant growth and development, especially during seed maturation reviewed in [1]. Mutant plants with altered ABA biosynthesis, perception or response have been crucial in deciphering the various components involved in ABA responses.

\footnotetext{
* Correspondence: adrian.cutler@nrc-cnrc.gc.ca

'Plant Biotechnology Institute, National Research Council of Canada, 110 Gymnasium Place, Saskatoon S7N OW9, Canada

Full list of author information is available at the end of the article
}

Recently, a family of 14 novel START domain proteins, named as PYR/PYL/RCARs (PYRABACTIN RESISTANCE/PYR1 LIKE/REGULATORY COMPONENT OF ABA RECEPTOR) has been identified as intracellular ABA receptors that interact with and inhibit several protein phosphatase 2Cs (PP2Cs) including ABA INSENSITIVE1 and 2 (ABI1, ABI2), HOMOLOGY TO ABI1 (HAB1), and PP2CA [2-4]. Such phosphatases are negative regulators of ABA signaling. A recent model for ABA signaling, based on several independent crystallographic studies for example [5], proposes that in the presence of $\mathrm{ABA}$, receptors of the PYR/PYL/RCAR family bind to PP2Cs which in turn release inhibition on a subfamily of SNF1-RELATED PROTEIN KINASE2 (SnRK2) kinases. These kinases then phosphorylate and subsequently activate transcription factors including $A B A$ RESPONSIVE ELEMENTS-BINDING FACTOR (ABF)/ ABA RESPONSIVE ELEMENTS-BINDING PROTEIN 
(AREB)-type bZIP TFs. ABFs in turn bind to ABAresponsive promoter elements (ABRE) to activate ABAresponsive gene expression.

The molecular basis of adaptive responses to abiotic stresses such as low temperature, desiccation and salinity, has been elucidated by identifying genes such as RESPONSIVE TO DEHYDRATION (RD), KYKNAINDUSOITU (KIN; Finnish for cold-induced), RESPONSIVE TO ABA (RAB), COLD-REGULATED (COR), LOW-TEMPERATURE-INDUCED (LTI), and DROUGHTINDUCED $(D I)$. Manipulation of many of these genes resulted in plants with improved tolerance to drought, salt, cold and freezing reviewed in [6]. Molecular and genetic studies suggest that ABA-dependent and -independent pathways operate in abiotic stresses and ABAdependent pathways are predominant in drought stress responses $[7,8]$.

Environmental factors such as drought, extreme temperature and pathogen infection as well as endogenous factors including age affect the onset and progression of leaf senescence reviewed in [9]. Unlike abscission and dehiscence, leaf senescence is a specialized form of programmed cell death, which is a genetically regulated process of slow cell death of the entire leaf and is preceded by the reallocation of nitrogen, phosphorus, and metals to other parts of the plant. An early manifestation of senescence in leaves is loss of chlorophyll. Leaf mesophyll cells start to senesce first, followed by other cell types, and exhibit an incoherent pattern of localized cell death, which eventually spreads to the whole leaf. Senescence is accelerated by ABA, ethylene, jasmonates (JAs), and salicylic acid (SA), and is delayed by cytokinins (CKs) and auxin reviewed in [9]. However, extensive cross talk among these signaling pathways during senescence complicates understanding of the initiation and progression of senescence. Therefore, key components in senescence signaling remain largely unknown.

Senescence is an important aspect of drought responses. Accelerated leaf senescence followed by leaf abscission is triggered by prolonged stress to reduce water loss, remobilize nutrients to young leaves and to enable survival of the plant $[10,11]$.

The MYB family TFs comprises around 180 genes in Arabidopsis and is the largest TF gene family reviewed in [12]. MYB proteins contain a conserved DNA-binding MYB domain of about 52 amino acids, and are classified into three subfamilies based on the presence of one, two or three MYB domains reviewed in [13]. The plant specific and largest MYB family consists of R2R3-type factors which contain two repeats and comprise 125 genes in Arabidopsis. R2R3-MYB genes are involved in various plant-specific processes such as regulation of secondary metabolism, modulation of development, determination of cell fate and identity and responses to environmental factors and hormone. The gene further characterized in this paper, AtMYBR1/MYB44 (R2R3 MYB) was weakly induced by $24 \mathrm{~h}$ treatment with $\mathrm{ABA}$ but strongly induced by the hyperactive ABA analog (+)-8' acetylene ABA (PBI425) [14]. Most ABA-regulated genes are similarly regulated by water stress, however $M Y B R 1$ was selected for further functional characterization because its expression was paradoxically repressed by drought and elevated by re-watering [8] suggesting a novel role in ABA signaling. Jung et al. [15] reported that over-expression of $M Y B R 1$ increased stress tolerance but unexpectedly repressed many known stress-related genes. Subsequent studies have revealed that this gene is regulated by a Mitogen-Activated Protein Kinase (MAPK) cascade. Following stress treatment, MITOGEN-ACTIVATED PROTEIN KINASE (MPK3) is activated and phosphorylates the bZIP TF VirE2-INTERACTING PROTEIN 1 (VIP1), which then rapidly activates the expression of $M Y B R 1$ and other stress genes through promoter binding [16]. Further studies have shown that MYBR1 interacts directly with, and is phosphorylated by, MPK3 at ser145 [17] and possibly ser53 [18] and that the ser145 phosphorylation is required for MYBR1 function [17]. In this study, we functionally characterized the AtMYBR1 TF by studying an Arabidopsis T-DNA insertion mutant mybr1 and overexpression lines of AtMYBR1 (OxMYBR1). We show that $M Y B R 1$ down regulates many ABA responsive genes including those involved in abiotic stresses and negatively regulates drought responses and senescence. Moreover, direct involvement of MYBR1 in early ABA signaling is suggested by our observation that MYBR1 protein interacts with PYL8, an ABA receptor.

\section{Results}

\section{AtMYBR1 represses genes induced by a hyperactive ABA analog}

We showed in a previous study that AtMYBR1 was induced weakly by (+)-ABA and more strongly by $24 \mathrm{~h}$ treatment with a hyperactive ABA analog PBI425 ((+)-8' acetylene $\mathrm{ABA}$ ) indicating $M Y B R 1$ is likely a component of the ABA signaling pathway [14]. It has been shown previously that $\mathrm{PBI} 425$ induces ABA responsive genes almost identically to the natural enantiomer S-(+)-ABA. However, because PBI425 is catabolized much less rapidly than (+)-ABA and accumulates to higher levels in plant tissue [14] it is an effective tool to study weak and transiently expressed ABA-responsive genes such as $A B I 1$, ABI2, LTI30, KNAT4 and MYBR1 itself [8,14,19]. Therefore we used PBI425 to define the role of MYBR1 in ABA signaling.

In addition to using PBI425 to study the function of AtMYBR1, we generated transgenic Arabidopsis $35 \mathrm{~S}_{\text {pro: }}$ : $M Y B R 1$ plants (OxMYBR1). After kanamycin selection, three lines with single inserts were selected and their 
homozygous progeny plants were grown as experimental materials. The level of overexpression of $M Y B R 1$ was 23-fold in gain-of-function OxMYBR1 line 42-6, 12-fold in line $31-3$ and 11-fold in line $1-7$ and was undetectable by qPCR in loss-of-function mybr1 [14]. To reduce the likelihood of identifying phenotypic artifacts due to mis-expression, the phenotypes of all the overexpression lines were compared for qualitative consistency throughout the experiments.

We compared gene expression in different genotypes using Arabidopsis oligoarrays representing a comprehensive set of approximately 26,000 expressed genes. The comparisons were: (i) genotype comparisons of untreated plants: OxMYBR1 (42-6) or mybr1 versus WT, (ii) genotype comparisons after PBI425 treatment: OxMYBR1 (42-6) or mybr1 versus WT, and (iii) effect of PBI425 treatments on each genotype: OxMYBR1, mybr1 and WT treated with PBI425 versus the same genotype without the treatment. The experimental design is illustrated in Additional file 1: Figure S1 online. Samples were treated with PBI425 for $24 \mathrm{~h}$ on the basis that the accumulation and effects of PBI425 on gene expression was maximum at $24 \mathrm{~h} \mathrm{[8].} \mathrm{The} \mathrm{total} \mathrm{numbers} \mathrm{of}$ differentially expressed genes are listed in Table 1 and the gene lists and data may be found in Additional file 2: Table S1. There were a total of 1507 differentially regulated genes from all comparisons. In the absence of PBI425 treatment, comparisons of OxMYBR1 or mybr1 vs. WT yielded a very small number of differentially regulated genes (Table 1). Treatment with PBI425 greatly increased numbers of differentially expressed genes and revealed differences between genotypes. Analysis of the direct effect of PBI425 on gene expression showed that $M Y B R 1$ represses expression of many genes induced by PBI425 in WT (and mybr1) in terms of both total numbers (Table 1) and expression ratios (Figure 1, Additional file 2: Table S1).

It is noteworthy that there were very few differentially regulated genes from comparisons of mybr 1 versus WT both with (12 genes) and without (2 genes) PBI425

Table 1 Number of significantly up- and down regulated genes using a threshold change in expression of 1.5 Fold and a P-value cut-off: $\leq \mathbf{0 . 0 5}$

\begin{tabular}{llll}
\hline Hybridization & Up & Down & Unchanged \\
\hline mybr1(PB|425) vs. mybr1 & 448 & 452 & 607 \\
WT(PBI425) vs. WT & 417 & 438 & 652 \\
OxMYBR1(PB|425) vs. OxMYBR1 & 180 & 246 & 1081 \\
OxMYBR1(PB|425) vs. WT(PB|425) & 88 & 420 & 999 \\
OxMYBR1 vs. WT & 35 & 86 & 1386 \\
mybr1(PBI425) vs. WT(PB|425) & 11 & 1 & 1495 \\
mybr1 vs. WT & 0 & 2 & 1505 \\
\hline
\end{tabular}

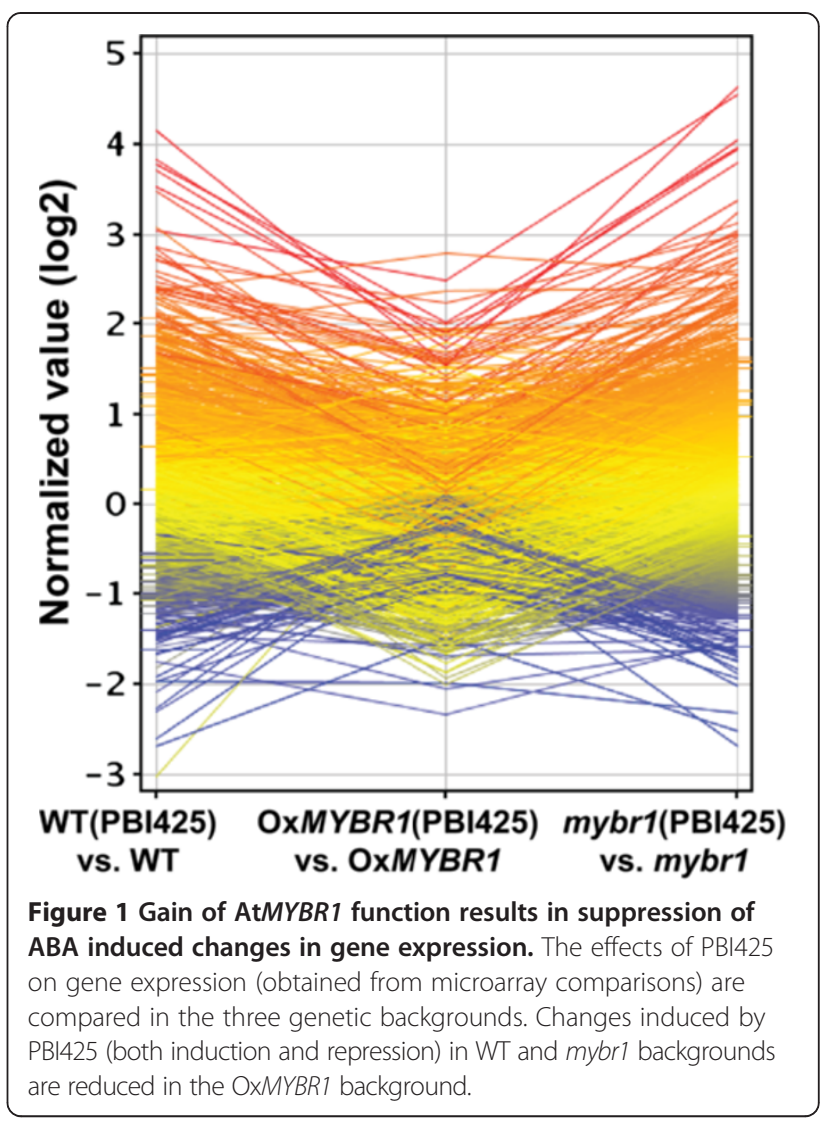

treatment. This suggests the likelihood that MYBR1 is functionally redundant with at least one other closely related gene. MYBR2 (MYB77) is reported as the closest homolog of $M Y B R 1$ based on sequence similarities in the C-terminal regions of the respective proteins and lack of homology with other MYB-type proteins [20]. MYBR2 has been reported to be involved in auxin signal transduction. MYBR2 over-expression results in reduced root and shoot growth, and root phenotypes in loss-function mybr2 lines varied with application of auxin [21]. However, its role in ABA responses and stress signaling or whether it acts cooperatively with $M Y B R 1$ remains unclear.

To investigate possible redundant functions of $M Y B R 1$ (MYB44) and MYBR2 (MYB77), gene expression analysis was performed using Agilent microarrays containing 44,000 Arabidopsis thaliana reporter sequences. Gene expression was compared between pairs of genotypes treated with PBI425 i) $m y b r 1$ versus $m y b r 2$ and ii) $m y b r 1$ versus mybr1xmybr2. The number of differentially expressed genes was 56 for mybr 1 vs $m y b r 2$ and 411 for mybr1 vs mybr1 x mybr2 (Additional file 2: Table S1). The increase in differentially expressed genes in the double mutant comparison suggests that MYBR1 and $M Y B R 2$ act in a synergistic manner However, only six out of 56 genes in the first comparison and 45 out of 411 genes in the second comparison were present in the above 
mentioned list of 1507 genes differentially expressed in all comparisons.

\section{AtMYBR1 represses many $A B A$ inducible stress genes}

Many stress genes that are highly induced by $A B A$, are repressed by MYBR1 (Figure 2). However, since MYBR1 did not appear to repress all PBI425 induced genes, we examined more closely the gene expression patterns affected by MYBR1 and PBI425. In this analysis, we added 32 statistically significant genes to the total gene list of Table 1 for detailed analysis and interpretation. These 32 genes were not listed in Table 1 since their fold change was below the 1.5 ratio threshold. However, changes in expression of these genes were either verified by qPCR and direct spot visualization in BASE or were present in

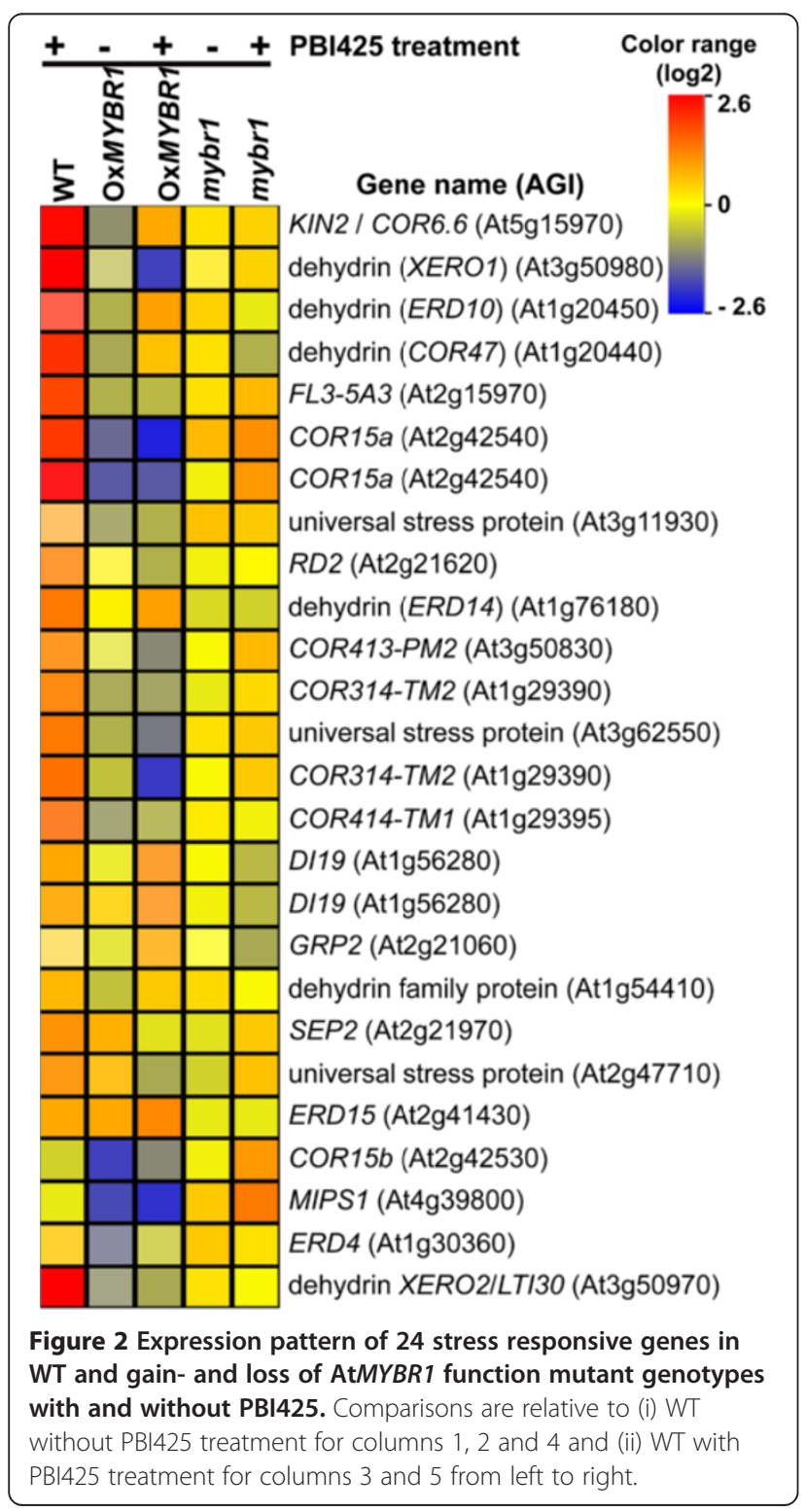

our comparative analysis of our microarray data with data published by van der Graaff et al. [22].

Several distinct gene regulation patterns (classes A - H) were identified by comparing PBI425 treatment and MYBR1 overexpression (Table 2) and the gene lists and expression data may be found in Additional file 3: Table S4. We excluded the comparison of mybr 1 vs. WT from Table 2 because of the small number of differentially expressed genes. Similarly, we also excluded microarray data of mybr2 from comparisons mybr1(PBI425) versus mybr2(PBI425) and mybr1(PBI425) versus mybr1xmybr2 (PBI425). Many well-characterized abiotic stress responsive genes were grouped in classes A, F and G, of which 278 genes (class $\mathrm{A}$, representing repression of $\mathrm{ABA}$ responses by $M Y B R 1$ ) were repressed by MYBR1, 112 genes (class F, ABA-like activation) were activated by MYBR1 and 254 genes (class G, representing ABA-specific activation) were unaffected by MYBR1. Therefore, MYBR1 represses a subset of about $43 \%$ of ABA-responsive genes. It has been suggested that stress responsive genes are under regulation by both ABA-dependent and ABA-independent pathways For a review see [7]. There are four DEHYDRATION RESPONSIVE ELEMENT BINDING PROTEIN1 (DREB1)/ C-REPEAT BINDING FACTOR (CBF) transcription factor genes that have been suggested to mediate ABA independent cold stress responses. Here, DREB1A/CBF3 was induced by PBI425 and repressed by MYBR1 suggesting that $D R E B 1 A / C B F 3$ is ABA-dependent.

Many senescence associated genes including SAG12/ 13/21/29/102, EARLY RESPONSIVE TO DEHYDRATION1 (ERD1) and APG8a are also grouped in class A (downregulated by MYBR1 but upregulated by PBI425). There were a total of 498 genes that were either upregulated (class F) or down regulated (class B) by MYBR1 in the same direction as ABA (PBI425). Among the downregulated genes were many associated with photosynthesis and biotic stresses. A total of 198 genes (classes D and H) were regulated by MYBR1, but unaffected by PBI 425 . Among these, several genes involved in jasmonate and auxin action were repressed.

Promoters of many genes associated with drought, cold stress and salinity contain ABA-responsive element (ABRE), ABRE binding factor (ABF) and dehydration responsive element (DRE) [23]. A search for statistically over-represented cis-acting motifs present in the promoters of classified genes sets in Table 2 was carried out against AGRIS and PLACE databases using the analysis tools in Athena [24] and the results are summarized in Additional file 1: Table S2. These promoter motifs mainly consisted of four groups. The majority of motifs are associated with ABA. Other motifs are related to stress, light regulation, gibberellins (GA) and circadian clock, suggesting cross talk between these pathways as was also observed previously [14]. There was a general similarity in 
Table 2 Combined effects of ABA (PBI425) treatment and MYBR1 overexpression on ABA-related gene expression

\begin{tabular}{llcccccc}
\hline Class & $\begin{array}{l}\text { Classification of gene } \\
\text { regulation type }\end{array}$ & $\begin{array}{l}\text { ABA in } \\
\text { WT bk }\end{array}$ & $\begin{array}{c}\text { ABA in } \\
\text { mybr1 bk }\end{array}$ & $\begin{array}{c}\text { ABA in } \\
\text { Ox bk }\end{array}$ & $\begin{array}{c}\text { MYBR1 Ox in } \\
\text { ABA/WT bk }\end{array}$ & $\begin{array}{c}\text { MYBR1 Ox in } \\
\text { WT bk }\end{array}$ & $\begin{array}{c}\text { Differentially } \\
\text { expressed } \\
\text { genes } \mathbf{N}^{\circ}\end{array}$ \\
\hline A & Repression of ABA & $\uparrow$ & $\downarrow$ & $\uparrow$ but lowered & $\downarrow$ & $\downarrow$ & 278
\end{tabular}

response

$\mathbf{1}^{\circ}$ metabolism (SUS3; BMY7; GOLS2; SIP2; ALDH7B4; AOX1A; LP1; LTP3/4; PLDS; POP2; LKR; CORI3) $\mathbf{2}^{\circ}$ metabolism (4CL1; CCOAOMT1; OMT1; ELI3-1(CAD); CPISCA; CUT1; PAP1); ABA (NCED4; ABF3; ABI1; HVA22D; PP2C); ethylene (ACO putative; MBF1C; EBF1); jasmonates (VSP1; VSP2); redox (CAT2; FSD1); abiotic stress (P5CS1; COR15a/15b; FL3-5A3; COR413-PM1; USPS; RCI2B; ERD4/10; COR47; XERO2; KIN2(COR6.6); DREB1A(CBF3); RD29B; RD29A(COR78/LTI78); RCI2A/2B; DI21: RD2/22; RAB18); GPX6; BGAL6; senescence (SAG12/13/21/29/102; ERD1; APG8a/8e); cell wall (XTH7; AGP12; EXLA1); GSTZ1; GSTU7/16; CYP89A5/A6; ACP5; VIF1; TFS (DREB1A; STO; STH3/LZF1; HAP5C; HB1/7/12; AGL2O(SOC1); MYB73; GT2; NFYC3); HAB1/2; LEA14; ACD1; RD26; COR413-PM2; COR414-TM1; COR314-TM2; signaling (ROP2; GDI1);

Photosynthesis (PSI; PSII; RBCS-1A/1B); GAPA-2; cell wall (CSLD5; FLA8; AGP11/ 13/16/20/21; EXPA1/8/10/15; XTH6); lipid (ACP1/3/4; NMT3; CLS; SLD1); NIA2; auxin (ILL2; responsive genes); DWF1; 40S and 60S ribosomal protein genes; biotic stress (DAD1/2; PDF1.1/1.2/1.2b/1.2c/1.3/2.2/2.3; T/R); senescence (SEN1); TFs (RAP2.4; COL5/15; GATA5; KNAT6; WUS; BET9); SNG1; cytokinin (ARR4); signaling (CAM3; CDPK6; CPK7; MKK5; GRF6):

C MYBR1 independent $A B A$ repression

D $\quad$ ABA independent repression

E Constitutive activation of ABA-repressed responses

F $\quad$ ABA-like activation

G MYBR1 independent ABA activation

$\downarrow$

(1)

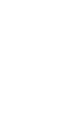

$-$

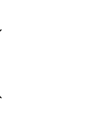

$\downarrow$

$\downarrow$

$\uparrow$

20

254

H ABA independent activation
112

Photosynthesis (PSII; RBCS-3B); cell wall (CSLC4; FLA9; EXPA3/5/6/11; PME1/3); lipid (FAD3/5/7; NMT2; ATS1); auxin (PIN4; IAA4; responsive genes); cytokinin (ARR5/7; IPT2); GA (GASA4); 30S, 40S, 505 and 60S ribosomal protein genes; abiotic/biotic stress (ERD3; PRS); tetrapyrrole biosynthesis; TFS (BEE2; PRE1; HB5); TCH3; ERD6; TIP2

171 myo-Inositol (MIPS1); cell wall (CESA1; AGP1/4/7/15/17; FLA1/2; PRP1; EXPA7; EXPB3; XTH9); lipid (FAD6; CER10); auxin (NIT1; ILL1; SHY2 (IAA3); ARF8); jasmonates (LOX2); abiotic stress (ERD4); TFS (NGA1; TRY; MYBL2); protein degradation (SCPL2; PREP1: MMZ1(UEV1A); UBC1; RHA2B: PAB2; PAC1; PAA1): signaling (GLR3.3; RAN3; ROP4; GRF12); transport (PIP3B; PIP2A; AAP2; CAXI; signaling (GLR3.3; RAN3; ROP4; GRF12); tran
TGD1; PATL1); PSII (PSBO-2; LHCB2.2/4.2);

TGD I; PATL 1); PSII (PSBO-2; LHCB2.2/4.2);

FAD2; SEX1; HMG1; NIT2; $\gamma$-VPE; senescence (SEN2 (CAT3)); abiotic/biotic stress (ERD14/15; HSP15.7-Cl; ERDJ2A; wound-responsive gene; PCC1); signaling (RD2O; TCH2; CAM1; RAB2; ELF4); PP2A-4;

myo-Inositol (MIOX1); major carbohydrate metabolism (APL3; SUS1; RCP1); fermentation (ADH; ALDH2B4); cell wall (FLA11; MERI5B; XTR3; EXT3); lipid (DGK1; LTP2; ACX1: MFP2; CUT1; CER1); abiotic stress (SEP2; KIN1; J8/20; ACD32.1; DI19; AOC1; RD22/26; ERD7); ABA (ABA1; CYP707A2); ethylene (ERF4); TFS (HAT2; DI19; AOC1; RD22/26; ERD7); ABA (ABA1; CYP707A2); ethylene (ERF4); TFS (HAT2;
HAP3; GBF3; KNAT4); protein degradation (RD21A; SCPL11; CLPXC; L1D; UBQ10; HAP3; GBF3; KNAT4); protein degradation (RD21A; SCPL11; CLPXC; L1D; UBQ1;
UBC28/30/32); signaling (RAB7B; RAN-1; MKKY; RAFL32; PAT1); DRM1; $\beta$-VPE; transport (AHA3; TMT2; SUC2; AAP1; NTP3; KUP11; GCN5; AATP1);

SON1; SFP1; TGG1; TGG2; WAK2; $\beta$-amylase; CSLG3; ACD6; CAM6; 
the presence of $A B A$ and stress motifs in genes that were regulated specifically by $A B A$ and those that were regulated in the opposite direction by MYBR1. No significant enrichment was detected in genes that were regulated specifically by $M Y B R 1$.

\section{AtMYBR1 reduces drought tolerance}

To define the function of MYBR1 during drought stress, plants were treated with $10 \%$ and $15 \%$ polyethylene glycol (PEG) for $5 \mathrm{~d}$. Consistent with the down regulation of the stress responsive genes (Figure 2), OxMYBR1 plants showed the highest degree of stress (leaf curling, bleaching) following PEG treatment and homozygous mybr1xmybr2 plants showed the least damage (Figure 3A). Subsequently, we found that OxMYBR1 rosettes lost water and chlorophyll faster than WT and loss-of-function mutants (Figure 3B and 3C). Therefore, consistent with the down regulation of stress responsive genes, the data suggests that OxMYBR1 plants transpired water faster and are consequently less drought tolerant than WT plants.

Drought stress was also imposed by withholding water for $18 \mathrm{~d}$ on seedlings from all available genotypes. Surprisingly, we recorded a $100 \%$ survival in all 10 replicates of OxMYBR1 (42-6) (Additional file 1: Figures S2A and S2B). Results obtained when drought stress was imposed by withholding water were opposite to what was observed following PEG mediated drought stress.

When performing the soil drying experiments above it was observed that, when plants from each genotype were provided with equal volumes of water, the OxMYBR1 soil dried more slowly than other genotypes. Therefore to investigate the contradictory results obtained by PEG and soil drying experiments, we conducted transpirational water loss assays on whole plants (Additional file 1: Figure S2C). In these experiments, soil water loss by evaporation was prevented so that plant water use could be

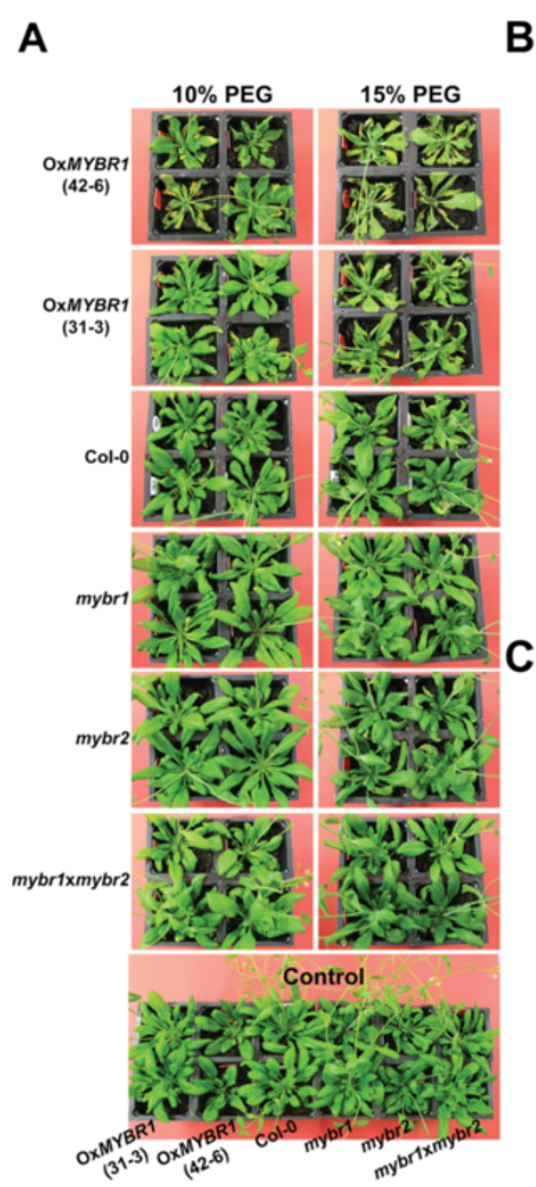

\section{B Detached Whole Rosette Leaf}

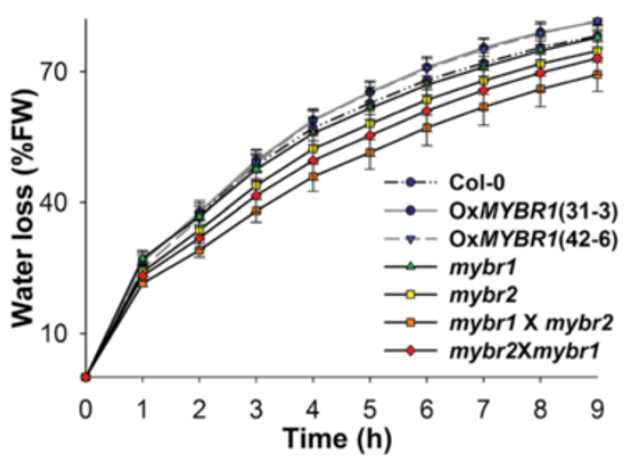

C Detached Whole Rosette Leaf

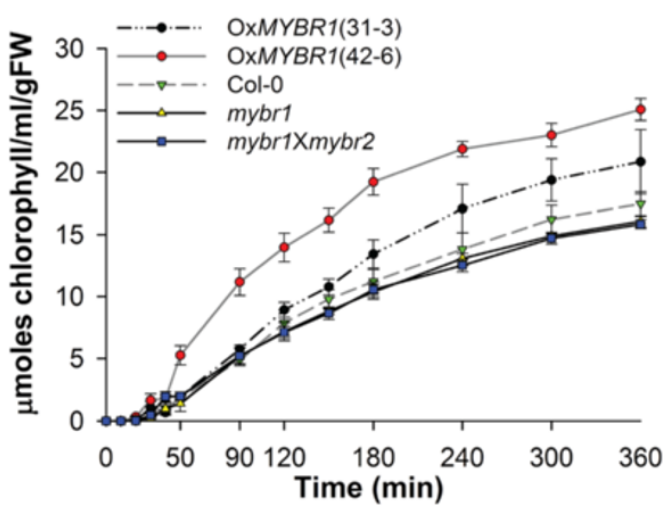

Figure 3 Gain of AtMYBR1 function results in reduced drought tolerance. (A) Reduced drought tolerance in the OXMYBR1 (35S pro:MYBR1) plants - lines 31-3 and 42-6. PEG at 10\% and 15\% concentrations was applied to $35 \mathrm{~d}$ old plants of OxMYBR1, mybr1, mybr2 and mybr1xmybr2 and WT (Col-0). Eight plants were used for each treatment. Pictures were taken $5 \mathrm{~d}$ after PEG treatment. (B) Detached whole rosette leaf water-loss from $20 \mathrm{~d}$ old plants. Transpirational water loss and standard error was calculated at each time point $(\mathrm{bar} ; \mathrm{n}=6)$. The P-value of two factor ANOVA is $4.7 \mathrm{E}-18$. (C) Rate of chlorophyll leakage from detached whole rosette leaf; standard error (bar; $n=6)$. 
monitored. The results showed that OxMYBR1 plants lost water slower than WT and mutants, which is contrary to what was observed in detached whole rosette leaves (Figure 3B). In other words, OxMYBR1 plants extracted and/or used less water from the soil than other genotypes, even though the transpirational capacity of the detached leaves was relatively higher. Therefore, we investigated the water conservation characteristic of OxMYBR1 further. We measured the soil water content after imposing an $8 \mathrm{~d}$ drought. Results confirmed that water uptake was less in OxMYBR1 than in WT and loss-of-function mutants during drought (Additional file 1: Figure S2D). Under normal (unstressed) growth conditions, OxMYBR1 plants grew more slowly than WT and loss-of-function mutants (Additional file 1: Figure S2A) and we show later that OxMYBR1 lines have shorter primary roots than other genotypes. Therefore, with respect to MYBR1 function, we conclude that reduced water use in whole OxMYBR1 plants in drying soil is not due to a genetically determined reduction in transpiration but is rather a consequence of lower biomass leading to slower depletion of soil moisture. The reduced growth rate of OxMYBR1 lines was also noted by Jung et al. [15] and in soybean by Seo et al. [25].

As an aside, we note that the residual water content of mybr2 material was slightly higher than the other lossof-function lines (Additional file 1: Figure S2D). This may be an indication that the functions of MYBR2 are not identical with those of $M Y B R 1$, as discussed later.

\section{MYBR $1_{\text {pro:GUS is expressed under abiotic stress and }}$ during senescence, mechanical wounding and floral organ abscission}

A $2.7 \mathrm{~kb}$ promoter fragment of MYBR1 including the $5^{\prime}$ UTR was fused to the $\beta$-GLUCURONIDASE (GUS) reporter gene $\left(M Y B R 1_{\mathrm{pro}}: G U S\right)$ and the expression of MYBR1 was examined histochemically. GUS staining was performed on homozygous $\mathrm{T}_{2}$ and $\mathrm{T}_{3}$ plants. In $13 \mathrm{~d}$ old seedlings (Additional file 1: Figure S3A), GUS expression driven by the MYBR1 promoter was observed in cotyledons and true leaves. In contrast to very high GUS expression in cotyledons, GUS expression was lower in younger true leaves relative to older leaves and was absent in the newly emerged leaves. Intriguingly, GUS expression was observed in patches in younger leaves and was absent around the vascular regions of both older and younger leaves hinting that MYBR1 could be involved in senescence since this pattern was reminiscent of the development of visible senescence in leaves reviewed in [9]. Under normal conditions, expression of GUS was also observed in hydathodes of all leaf margins as well as embryo, suspensor, endosperm, root, stigma, sepal, petal and anther filament but was absent in stem, cauline leaf, anther, silique and testa (Figure 4A, C, D and E and Additional file 1: Figure S3). GUS expression was observed in embryo and endosperm dissected from siliques at developmental stages from 6-18 DPA as well as from dry and imbibed (30 min - $99 \mathrm{~h}$ ) mature seeds (Additional file 1: Figure S3B and S3C). The intensity of GUS staining increased with development in embryos but remained constant in endosperm except at 6 DPA when the GUS expression was lower. GUS expression was high and remained constant in embryos collected from dry seeds and seeds imbibed up to $24 \mathrm{~h}$ but declined subsequently. GUS expression in endosperm of dry and imbibed seeds remained high.

Water stress significantly reduced GUS expression driven by the MYBR1 promoter in leaves but not in roots relative to control (Panel A in Figure 4). Drought induced reduction of MYBR1 expression is consistent with the reduced expression of $M Y B R 1$ under drought treatment observed by Huang et al. [8].

GUS reporter activity was rapidly and strongly induced immediately after mechanical wounding of leaves (Panel F in Figure 4). Similar high MYBR1 expression was also observed at the abscission zone (AZ) on the pedicel following sepal, petal, and anther filament abscission (Figure 4D and $4 \mathrm{E}$ ). Prior to the abscission of floral organs (Figure 4B and $4 \mathrm{C}$ ), no GUS expression was visible on the pedicel. However, we did not observe either acceleratedor delayed/abolished floral organ shedding in MYBR1 loss-and gain-of-function mutants, suggesting that the high GUS expression at the AZ is due to the wounding response.

\section{AtMYBR1 delays leaf senescence}

Early in leaf senescence chloroplasts disassemble with subsequent degradation of chlorophyll and visible leaf yellowing. To further investigate the role of MYBR1 in leaf longevity, detached rosette true leaves numbers 3-6 (counted by order of emergence), from $30 \mathrm{~d}$ old soil grown plants were incubated in buffer as described [26] in two different sets. Leaves were photographed and the chlorophyll content was quantified on $0 \mathrm{~d}$ for one set and after 6-7 d of dark-induced senescence treatment for the other set. In freshly harvested leaves, the chlorophyll content was higher in one line of OxMYBR1 (\#42-6) and two reciprocal double mutants of mybr 1 and mybr2 than the rest of the genotypes (Figure 5B). Following darkinduced senescence, OxMYBR1 lines showed increased leaf longevity and slowed chlorophyll degradation relative to WT leaves (Figure 5A and 5C). Interestingly, increased leaf longevity in OxMYBR1 lines was in contrast to that in mybr1 and reciprocal mybr1 \& mybr2 mutants which exhibited early leaf senescence and accelerated chlorophyll degradation relative to WT (Figure 5A and 5C) although the chlorophyll content before treatment was relatively high in mybr 1 and mybr2 double mutants (Figure 5B). 

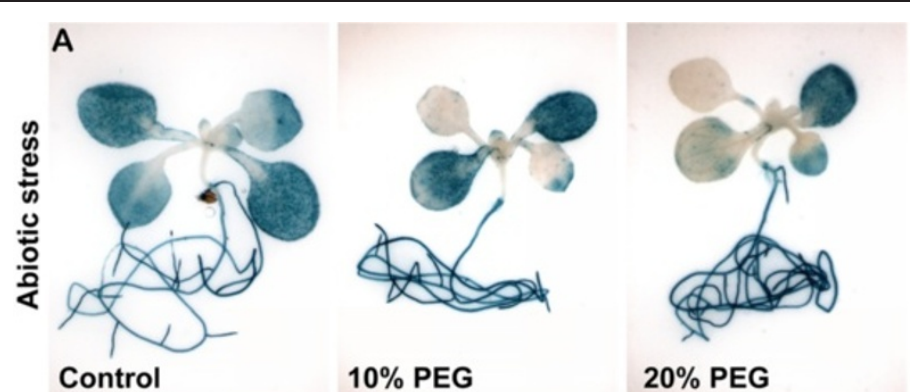

$10 \%$ PEG
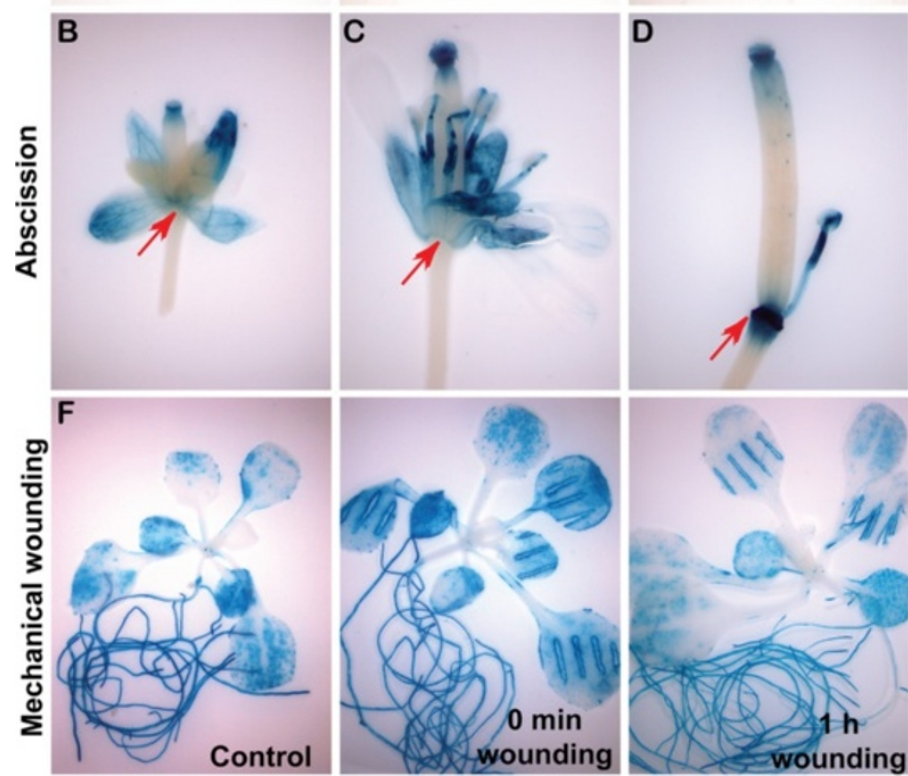
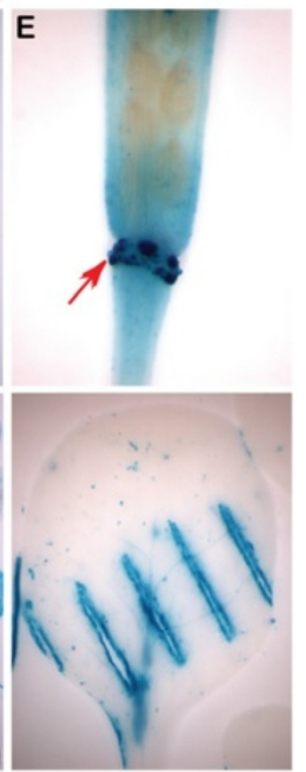

Figure 4 AtMYBR1 promoter drives GUS expression during abiotic stress, floral organ abscission and mechanical wounding. Histochemical localization of GUS activity was performed by staining with X-gluc for different time intervals as described below and in Methods. Panel (A) Significant reduction of GUS expression in leaves after drought stress. However, intense GUS expression in roots was similar to control. (B) A closed flower was opened manually by forceps before GUS staining was performed. (C) A fully opened flower. (B) and (C) No GUS expression was found on pedicel at the point where the sepal, petal and anther join (shown by arrows). (D) and (E) In contrast, intense GUS expression was observed at pedicel connecting sites of floral organs after abscission (arrowheads). (E) A silique fully abscised with floral organs, was stained briefly ( 2 h) for GUS. Magnification of the connections between floral organs and pedicel shows GUS expression only at attachment sites on pedicel. Panel (F) Leaves were wounded with hemostats. Intense MYBR1 promoter driven GUS expression was observed around the wound relative to control.

Indeed, among the eight genotypes, leaves of mybr 1 E mybr2 were least green following senescence treatment and showed the fastest chlorophyll breakdown (Figure 5A and 5C).

To further investigate the role of MYBR1 in leaf senescence, excised leaves of $37 \mathrm{~d}$ old transgenic $M Y B R 1_{\text {pro: }}$ : GUS were stained for GUS before and after dark induced senescence (Figure 5D). In untreated plants, GUS staining was strong in older leaves and was absent in younger leaves and in vascular region. GUS staining became weaker overall in dark-treated leaves relative to fresh leaves but among the dark treated leaves, there was more GUS staining in senescent, yellow leaves (indicated by asterisks) than green ones, further suggesting that MYBR1 plays a role during leaf senescence.

\section{AtMYBR1 regulates the expression of senescence genes}

To investigate whether MYBR1 regulates the expression of senescence genes, we compared the differentially expressed gene lists of Table 2 with microarray data obtained by van der Graaff et al. [22] on various stages and types of leaf senescence (NS: developmental senescence; DIS: darkening-induced senescence; DET: senescence in dark-induced detached leaf). The number of common genes between the two microarray analyses is 852 (Additional file 3: Table S4) which covers 52\% of our differential gene list. The increase of MYBR1 induction with the progression of senescence is high in NS, low in DIS and DET whereas MYBR2 induction is slightly increased in sink-to-source transition (5 week) stage of NS [22].

The regulation of these 852 common genes by ABA, $M Y B R 1$ and senescence revealed the interaction between 


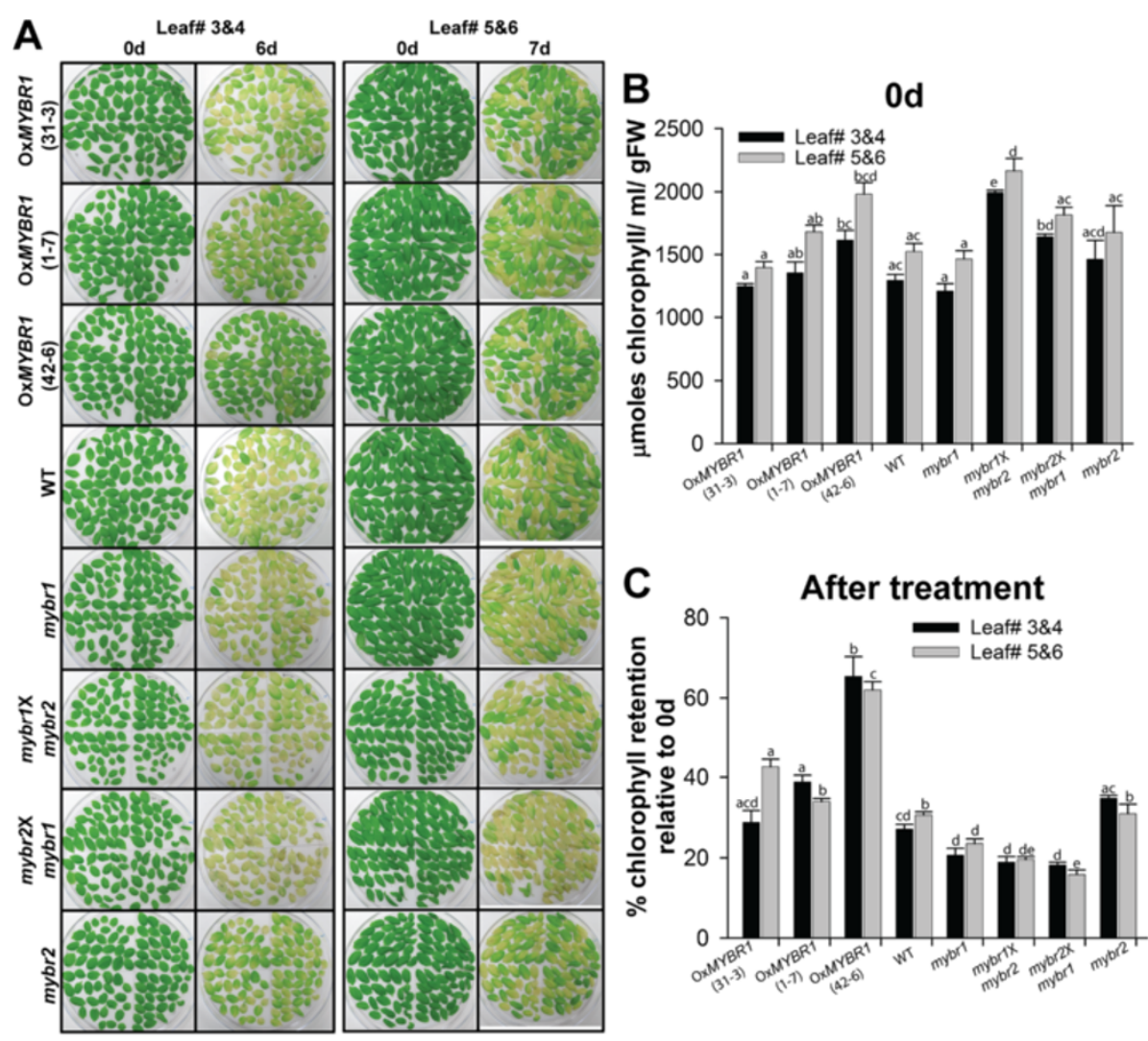

\section{Promoter MYRR $1-G U S^{-G}$}

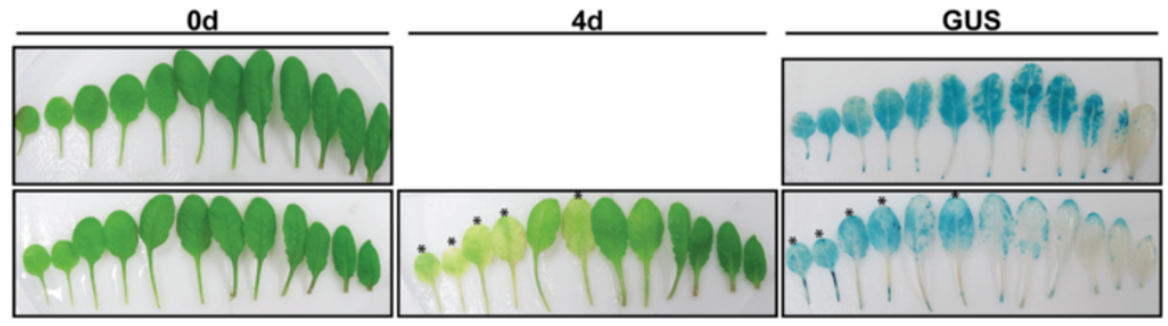

Figure 5 Effect of MYBR1 on leaf senescence in a detached leaf assay. (A) True leaves numbers 3-6 were harvested from $30 \mathrm{~d}$ old soil grown plants and incubated on filter paper wetted with 3 mM MES buffer ( $\mathrm{pH}$ 5.7). Leaves 3 and 4 were photographed after $6 \mathrm{~d}$ treatment and leaves 5 and 6 were photographed after $7 \mathrm{~d}$ treatment. Leaves from OXMYBR1 plants of three independent lines (\#31-3, 1-7 and 42-6) showed delayed senescence relative to other genotypes and leaves from mybrl and double mutant plants exhibited premature leaf senescence relative to leaves from WT (leaves 5 and 6) and OxMYBR1 plants (all leaves). Two sets of experiment were carried out as above (A) in four replicates and 12 plants in each replicate. Statistical significance was determined using one-way ANOVA with Tukey using the statistical software ' $R$ ' $(P<0.05)$. (B) In one set of experiments, chlorophyll was extracted and measured on $0 \mathrm{~d}$. The chlorophyll content was higher in one line of OxMYBR1 (\#42-6) and reciprocal double mutants of mybr1 and mybr2 than other genotypes. (C) In another set, chlorophyll was extracted and measured on $6 \mathrm{~d}$ for leaves 3-4 and on 7d for leaves 5-6 and the percentage chlorophyll retention was calculated relative to $0 \mathrm{~d}$ from (B). Chlorophyll retention was generally higher in OXMYBR1 genotypes. (D) Using three independent homozygous MYBR1 pro:GUS lines (\#5-1, 7-6 and X1-4), experiments were carried out as above (A) in two replicates. All leaves from each plant were harvested. GUS staining was performed on $0 \mathrm{~d}$ (untreated) and after $4 \mathrm{~d}$ of dark induced senescence. Asterisks indicate yellow leaves before and after GUS staining. After the treatment, GUS staining was higher in senescent leaves, but in green leaves was lower than corresponding control leaves.

ABA and MYBR1 during senescence (Additional file 1: Table S5 and Additional file 3: Table S4 online). Out of 165 genes in class $\mathrm{A}$ (Table 2, repression of ABA response), $88 \%$ were induced by both ABA and NS but repressed by $M Y B R 1$, clearly demonstrating that MYBR1 is a suppressor and $A B A$ is an activator of leaf senescence. Furthermore, out of 261 genes in class C (MYBR1 independent $A B A$ repression), 95-96\% genes are downregulated by NS, DIS and DET and from 146 genes in class G (MYBR1 independent ABA activation), $86 \%$ are 
upregulated by NS in the same direction as by $\mathrm{ABA}$ showing a significant role of $A B A$ in leaf senescence. Of a large number of common genes (161; class B), 8993\% are downregulated by ABA, MYBR1 and senescence and many of these genes are involved in protein synthesis (23 ribosomal protein genes), photosynthesis (31 genes), auxin responses (6 genes) and biotic stress (5 genes).

Furthermore, we performed QRT-PCR on senescence marker genes in rosette leaves numbers 3 and 4 in all the genotypes (primers for QRT-PCR are listed in Additional file 1: Table S3). Consistent with our detached leaf senescence analysis (Figure 5), accelerated leaf senescence of mybr1xmybr2 was associated with upregulated expression of SAG12, SAG29 and SENESCENCE4 (SEN4) relative to all other genotypes (Figure 6). SAG29 expression was $70( \pm 8.5)$ fold higher in mybr1xmybr2 relative to WT. The expression of SAG12, SAG29, SEN1 and SEN4 were downregulated in OxMYBR1 relative to WT, consistent with the senescence-suppressing effect of MYBR1. Changes in expression of these genes in the single mutants mybr 1 and mybr2 were generally small. However, expression of SAG21 and SEN1 did not show a reciprocal relationship between gain and loss of function genotypes. Nevertheless, it is apparent that MYBR1 negatively regulates senescence based on 3 of the 4 marker genes.

Protein degradation occurs during senescence via different pathways such as autophagy (APG) and the $26 \mathrm{~S}$ proteosome and components of both pathways were transcriptionally activated during senescence [22,27]. Our differential gene list contains 87 genes involved in protein degradation pathways (Additional file 3: Table S4). Here we report the repression of 44 genes by OxMYBR1, where 31 genes encode components of E2 (ubiquitin-conjugating protein (UBC)), E3 ubiquitin-ligase complex and 20S core particle of $26 \mathrm{~S}$ proteosome and three genes are APG8al $8 f / 8$ e. Interestingly, only three genes in the ubiquitin pathway - PHD finger family, EARLY-RESPONSIVE TO DEHYDRATION 16/UBIQUITIN ETENSION PROTEIN 1 (ERD16/UBQ1) and SUPPRESOR OF NIM1-11 (SON1) were upregulated by OxMYBR1. SON1 is an F-box protein component of E3-ubiquitin ligase complex which negatively regulates, through the ubiquitin-proteosome pathway, a novel defense response that is independent of systemic acquired resistance [28]. On the other hand, 45 genes involved in protein degradation were activated by ABA in both WT and mybrl, and many of them are also upregulated by NS, DIS and DET [22].

\section{Content of endogenous cytokinins and jasmonic acid}

To investigate the role of MYBR1 in relation to hormonal pathways, endogenous hormone levels were measured quantitatively by LC-MS/MS in rosette leaves numbers 3 and 4 of three weeks old plants (Figure 7). trans-Zeatin $(\mathrm{t}-\mathrm{Z})$ and $\mathrm{N}^{6}-\left(\Delta^{2}\right.$-isopentenyl) adenine (iP) and their sugar derivatives are the major cytokinins (CKs) in Arabidopsis [29]. Levels of several CKs were significantly increased in OxMYBR1 relative to other genotypes. On the other hand, JA was significantly higher in mybr1 x mybr2 relative to other genotypes. This suggests that suppression of leaf senescence by MYBR1 is associated with inis associated with higher JA.

In our microarray data, OxMYBR1 (comparison: OxMYBR1 treated with PBI425 versus untreated OxMYR1) downregulated ARR4 (Table 2), a transcriptional repressor of CK signaling [30]. However, the down regulation of ARR4 by ABA (Table 2) and senescence [22] is

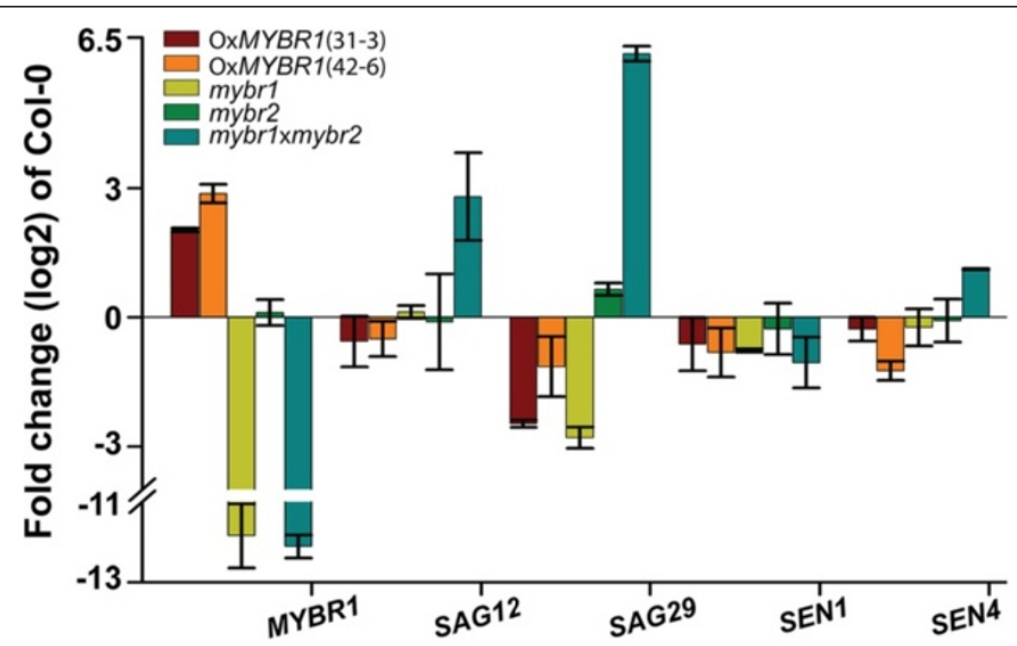

Figure 6 MYBR1 and MYBR2 regulate expression of some senescence-related genes. QRT-PCR was performed on total RNA extracted from rosette leaves numbers 3-5 of $21 \mathrm{~d}$ old soil grown plants of WT (Col-0), gain- and loss-of MYBR1 function as well as mybr2 and double mutant mybrixmybr2. Standard error $(n=2)$ of biological repeats are indicated. 


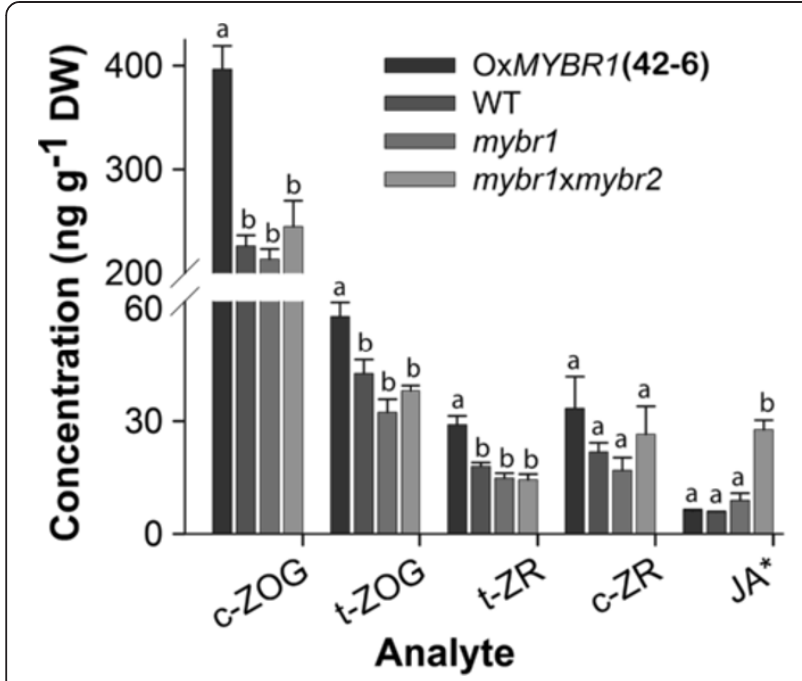

Figure 7 Endogenous levels of CKs and JA in rosette leaves numbers $3-5$ of $25 \mathrm{~d}$ old soil grown plants of WT, gain- and loss-of MYBR1 function and double mutant mybr1xmybr2. Leaves were harvested from at least 8 plants per replicate per compound measured. Standard error was calculated from each hormone $(n=4)$. CKs measured include: $c-Z O G$, cis-zeatin-O-glucoside; $\mathrm{t}-\mathrm{ZOG}$, trans-zeatin-O-glucoside; $\mathrm{t}-\mathrm{ZR}$, trans-zeatin riboside; $\mathrm{c}-\mathrm{ZR}$, cis-zeatin riboside. $\mathrm{JA}^{*}$ : Concentration was measured as $\mathrm{ng} \mathrm{g}^{-1} \mathrm{FW}$.

contradictory and may be due to a feedback effect. Furthermore, we did not detect differential expression of genes involved in CK metabolism but a posttranscriptional regulatory effect of $M Y B R 1$ on expression of these genes cannot be ruled out.

OxMYBR1 leaves contained similar levels of ABA and its metabolite dihydrophaseic acid to those measured in $\mathrm{WT} / m y b r 1$ and mybr1xmybr2. Levels of SA and IAA were also not significantly altered. It is surprising that ABA levels remained constant despite the strong effect of AtMYBR1 overexpression on ABA responses. To investigate further, we performed qRT-PCR (primers for QRT-PCR are listed in Additional file 1: Table S3) on six ABA downstream effectors; $A B I 5, E E L$ and $A B F 1 / 2 / 3 / 4$ in plants of OxMYBR1 (lines 42-6 and 31-3), WT, mybr1/2 and mybr $1 \times$ x mybr2 (Additional file 1: Table S3). No significant differences of expression of these genes were evident among the genotypes tested.

\section{MYBR1 mis-expression affects leaf and root morphology}

We examined the roots of gain-and loss of $M Y B R 1$ function genotypes (Figure 8). Primary roots of OxMYBR1 lines were drastically shorter, whereas those of mybr1 were notably longer relative to WT. This shorter root phenotype of OxMYBR1 may contribute toward reduced water uptake in the OxMYBR1 lines as noted earlier, and may help explain the differences between results of PEG treatments and soil drying experiments described above.

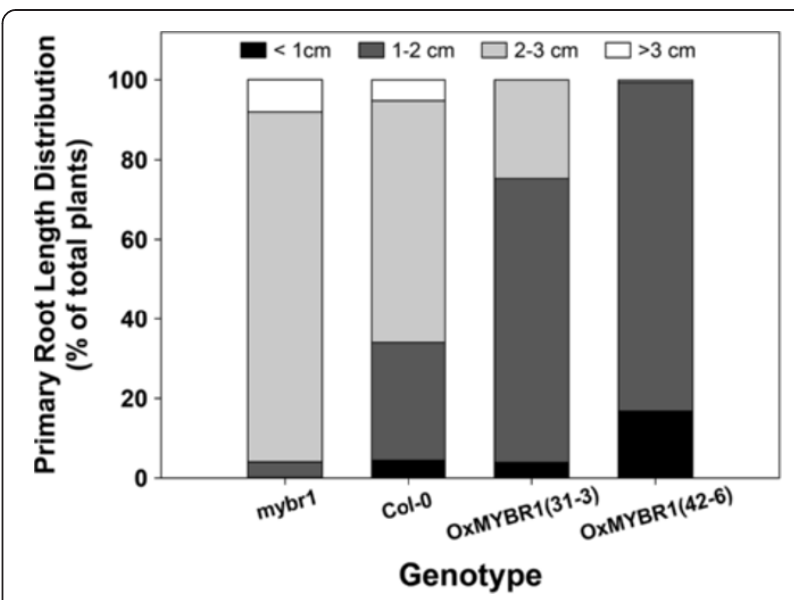

Figure 8 Primary root lengths of MYBR1 genotypes. Primary roots were measured in soil-grown 8 day old plants. The results are expressed as the percentage of roots of each genotype that fell within the four indicated length ranges. For each genotype, the total number of measured roots and the total range of lengths in $\mathrm{cm}$ were: OXMYBR1 42-6, 380 (range 0.54-2.12); OxMYBR1 31-3, 203 (range 0.71-2.71); Wild type, 270 (range 0.62-3.57); mybr1, 372 (range 1.22-3.59).

The Arabidopsis mutant amp1, with a high level of endogenous CKs, had increased numbers of rosette leaves [31]. Conversely, plants overexpressing catabolic CK oxidases had fewer leaves than WT plants [32]. We counted rosette leaves in seedlings of two lines of OxMYBR1, WT and mybr1. Seedlings of OxMYBR1 lines had consistently more rosette leaves relative to other genotypes and double mutant mybr1xmybr 2 had fewer leaves relative to all other genotypes (Figure 9). Differences between genotypes are slightly enhanced by ACC treatment. Using two way ANOVA, there is a consistent, statistically significant difference in leaf number between mybr 1 x mybr2 and the OxMYBR1 lines but no significant difference between control and ACC treatments.

\section{MYBR1 physically interacts with PYL8 and INO}

Further information on the mechanistic role of MYBR1 in signaling was obtained by identifying protein-protein interactions using the yeast 2-hybrid system. Initially PYR1-LIKE8 (PYL8) and INNER NO OUTER (INO) proteins were identified as interacting with MYBR1 by screening an Arabidopsis cDNA library made from different stages of vegetative and floral tissues with a full length MYBR1 fused to the DNA-binding (BD) domain of the yeast GAL4 protein. It has been shown by many groups that the 14 members of the PYR/PYL/RCAR family are intracellular ABA receptors that interact with and inhibit several PP2C-type protein phosphatases including $\mathrm{ABI} 1, \mathrm{ABI} 2, \mathrm{HAB} 1$ and PP2CA [2-4]. INO encodes a YABBY-type TF and is required for both polarity determination and outer integument initiation in ovule development [33]. 


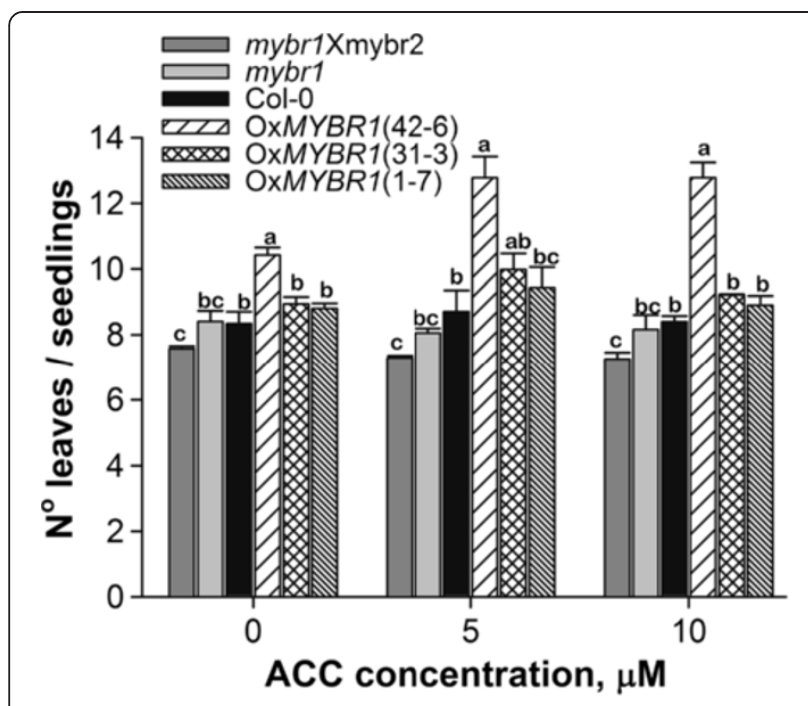

Figure 9 Leaf number in MYBR1 genotypes. Seeds from WT, mybr1, mybr1xmybr2 and three lines of OXMYBR1 (42-6, 31-3 and 1-7) were germinated on MS plates and $8 \mathrm{~d}$ old seedlings were transferred to fresh plates without and with 5 and $10 \mu \mathrm{M} \mathrm{ACC}$. Leaf number was counted $17 \mathrm{~d}$ after transfer. Control and ACC treated experiments were conducted in four replicates and each replicate contained four seedlings per genotype. The experiment was repeated three times. Standard error was calculated at each time point $(n=12)$. Two-way ANOVA resulted in significant differences between genotypes but no significant difference between control and ACC treatments. There were increased numbers of rosette leaves in OXMYBR1 lines relative to other genotypes (especially line 42-6) and a significantly reduced number of leaves in the mybrl and mybr2 double mutant relative to most other genotypes with and without ACC. However, ACC treatment qualitatively enhanced differences between genotypes.

MYBR1 interaction with PYL8 encouraged us to subsequently fuse all 14 PYR/PYL/RCAR genes as well as INO and $M Y B R 2$ to the transcription-activation (AD) domain of the yeast GAL4 protein. Interestingly, only PYL8, INO and MYBR2 interacted with MYBR1 (Figure 10). Interaction of MYBR1 and PYL8 was confirmed by three independent experiments using a more stringent screening of positive clones on four drop out media (SD/-Leu/-Trp/His/-Ade) in the presence of antibiotic Aureobasidin A. Furthermore, we also fused the full length MYBR2 to the $\mathrm{BD}$-domain and found that it also interacts only with PYL8 out of the 14 PYL family members. But MYBR2 showed no interaction with INO which suggests that, despite their shared roles in stress response and senescence, MYBR1 and MYBR2 have some non-redundant functions. In Figure 10, yeast colonies resulting from interaction of AD-pGADT7, -MYBR2, -PYL8, and -INO with BDMYBR1 were smaller compared to those with BDMYBR2 and BD-pGBT9. It should be noted that we observed slight autoactivation and notable toxicity/reduced cell growth from high expression of MYBR1 using the pGBKT7 plasmid and hence used the lower expressing plasmid pGBT9.
Next, we examined whether the protein-protein interactions described above are affected by phytohormones (Additional file 1: Figure S4). In addition, MYBR2 is reported to modulate auxin signaling [21] and therefore we also tested inhibitors of auxin signaling (PCIB) and transport (TIBA and NPA). However, the above interactions were constitutive and not affected by phytohormone additives.

We performed bimolecular fluorescence complementation (BiFC) assays in Nicotiana benthamiana leaf epidermal cells to independently verify the interactions of PYL8 with MYBR1 and MYBR2 and of MYBR1 with MYBR2. It has been shown previously that PYL8 and MYBR1 are localized in the nucleus [15,34] and interaction between MYBR2 and ARF7 occurs in the nucleus [21]. All interactions of PYL8 with MYBR1 and MYBR2 and of MYBR1 with MYBR2 are high with consistent fluorescent signal (Figure 9). Yeast two-hybrid and BiFC approaches confirmed the interactions of PYL8 with MYBR1 and MYBR2 and between MYBR1 and MYBR2 and showed that PYL8 may modulate the binding of MYBR1 and MYBR2 to DNA and/or that both MYBR1 and MYBR2 may modulate PYL8 function. The interaction of MYBR1, 2 with only PYL8 but not with other members of PYR/PYL/RCAR family suggests that these interactions define very specific functional roles.

\section{Discussion}

We previously identified $M Y B R 1$ as a weakly ABA responsive gene [14] and here we provide evidence that it is a repressor of ABA signaling during seedling growth, drought and senescence. It is now clear that MYBR1 is part of the ABA/abiotic stress response and wounding/ abscission response networks, both of which involve senescence responses. MYBR1 acts as a negative regulator (feedback repressor) of responses to stress, wounding and abscission in favor of normal growth and development. $M Y B R 1$ is by no means unique in its ability to negatively regulate $\mathrm{ABA}$ and stress responses. Other examples include the AP2 domain TFs ABA REPRESSOR1 (ABR1) [35] and ETHYLENE RESPONSE FACTOR7 (ERF7) [36] and the homeodomain protein $H B 6$ [37].

Our original observations that MYBR1 was induced by PBI425, induced weakly by ABA, repressed by drought and paradoxically induced by rewatering after drought stress $[8,14]$ have been confirmed and can now be rationalized. Under non-stressed conditions, ABA treatment produces unnecessary stress responses and MYBR1 induction blocks these responses to restore normal patterns of gene expression. Under water stress conditions MYBR1 is not expressed, allowing the full effects of ABA to be manifested and allows adaptive responses to be maintained during drought stress. On recovery from stress, MYBR1 expression leads to repression of ABA responses 


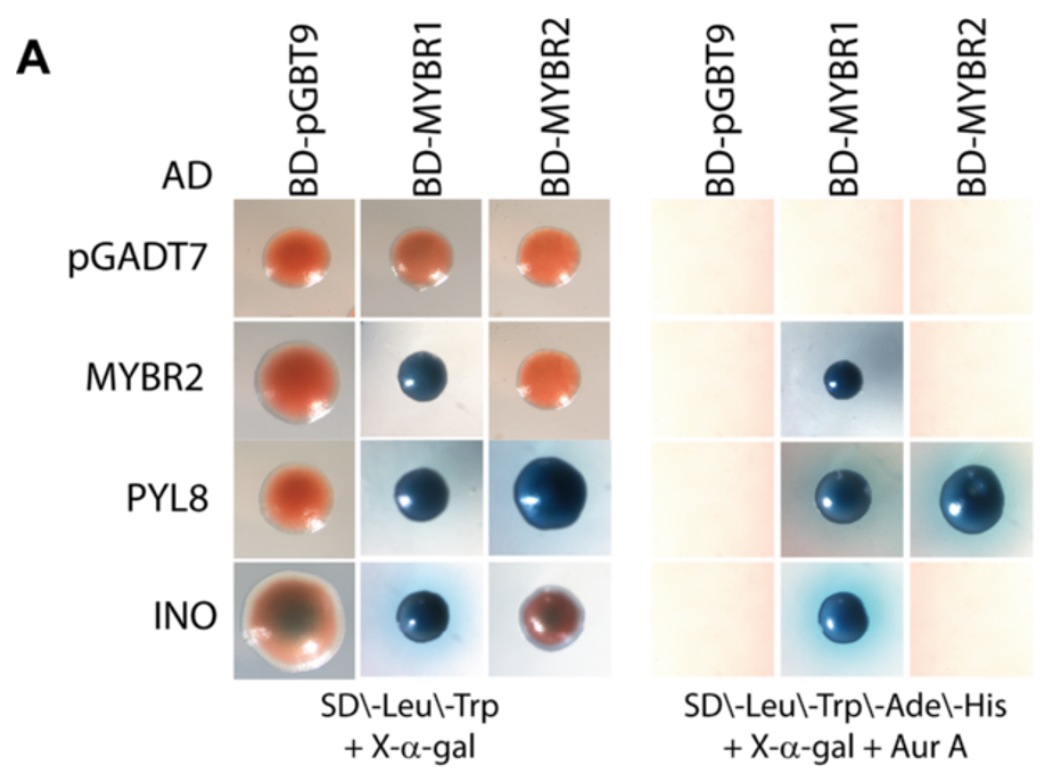

B

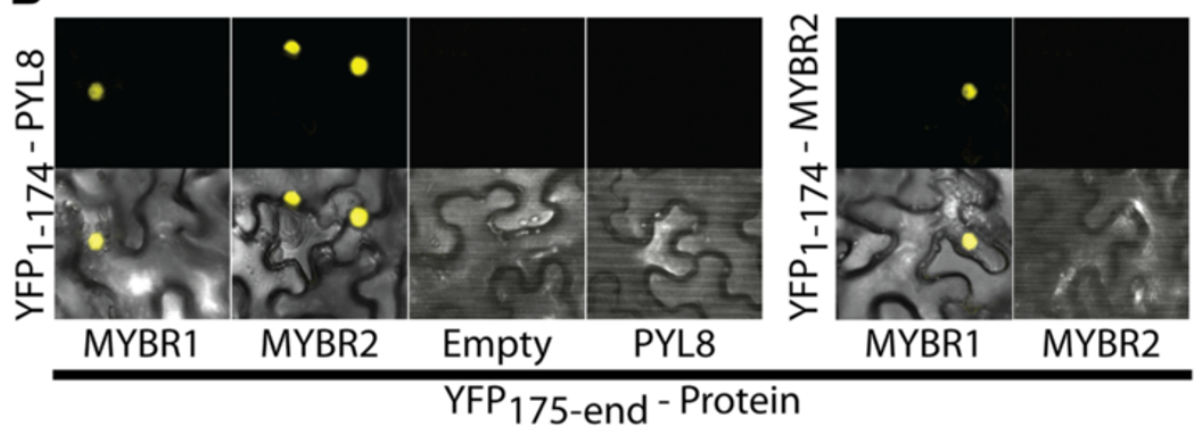

Figure 10 Physical interaction of MYBR1 and MYBR2 with PYL8, MYBR2 and INO as determined by the yeast two-hybrid method and BiFC. (A) Blue color from galactosidase activity indicates interaction between proteins produced from the bait (top row) and prey (vertical axis) vectors. (B) BiFC of the interactions of PYL8 with MYBR1 and MYBR2 and of MYBR2 with MYBR1 in N. benthamiana leaf epidermal cells. Top panel shows the signal from eYFP, reconstituted from YFP1-174 aa -PYL8 and YFP175-end aa -MYBR1 \& MYBR2 and from YFP1-174 aa -MYBR2 and YFP175-end aa -MYBR1. Bottom panel presents eYFP and transmitted light detector signals.

that are no longer required. The regulation and effects of $M Y B R 1$ are summarized in Figure 11.

Although induced senescence during prolonged drought has survival value by conserving water and nutrients [11], there are clearly finely balanced advantages and disadvantages to irreversible loss of vegetative matter. In fact, by introducing a novel feedback mechanism to suppress drought induced senescence in tobacco, Rivero et al. [10] demonstrated striking beneficial effects, suggesting that, in a crop plant context, induced senescence can be disadvantageous. Therefore, it seems that MYBR1 is a component of an endogenous homoeostatic mechanism to balance growth, high seed production and risk of death versus senescence, survival and minimal seed production. Given that senescence of older leaves is a normal stage of leaf development, MYBR1 appears to also play a role in determining the normal length of the leaf adult phase.
Senescence induces protein degradation pathways [22,27,38] and the effects of MYBR1 are associated with reduced/delayed expression of ubiquitin- and autophagy mediated protein degradation and increased production of CKs. Previous studies have associated droughtinduced leaf senescence with reduced CKs [11] and increased CK biosynthesis blocks leaf senescence [39]. Higher levels of CKs, reduced primary root growth and more adult leaves in OxMYBR1 lines are also consistent with increased CK effects. However, there are other hormonal interactions. MYBR1 appears to repress jasmonate effects - which likely also contributes to suppression of wounding responses. Jung et al. [40] demonstrated that MYBR1 was induced by jasmonate and also showed that jasmonate responses were repressed. More recently Shim et al. [41] show that MYBR1 represses JA defense responses and activates salicylic acid-mediated defenses via WRK70 leading to enhanced responses to 


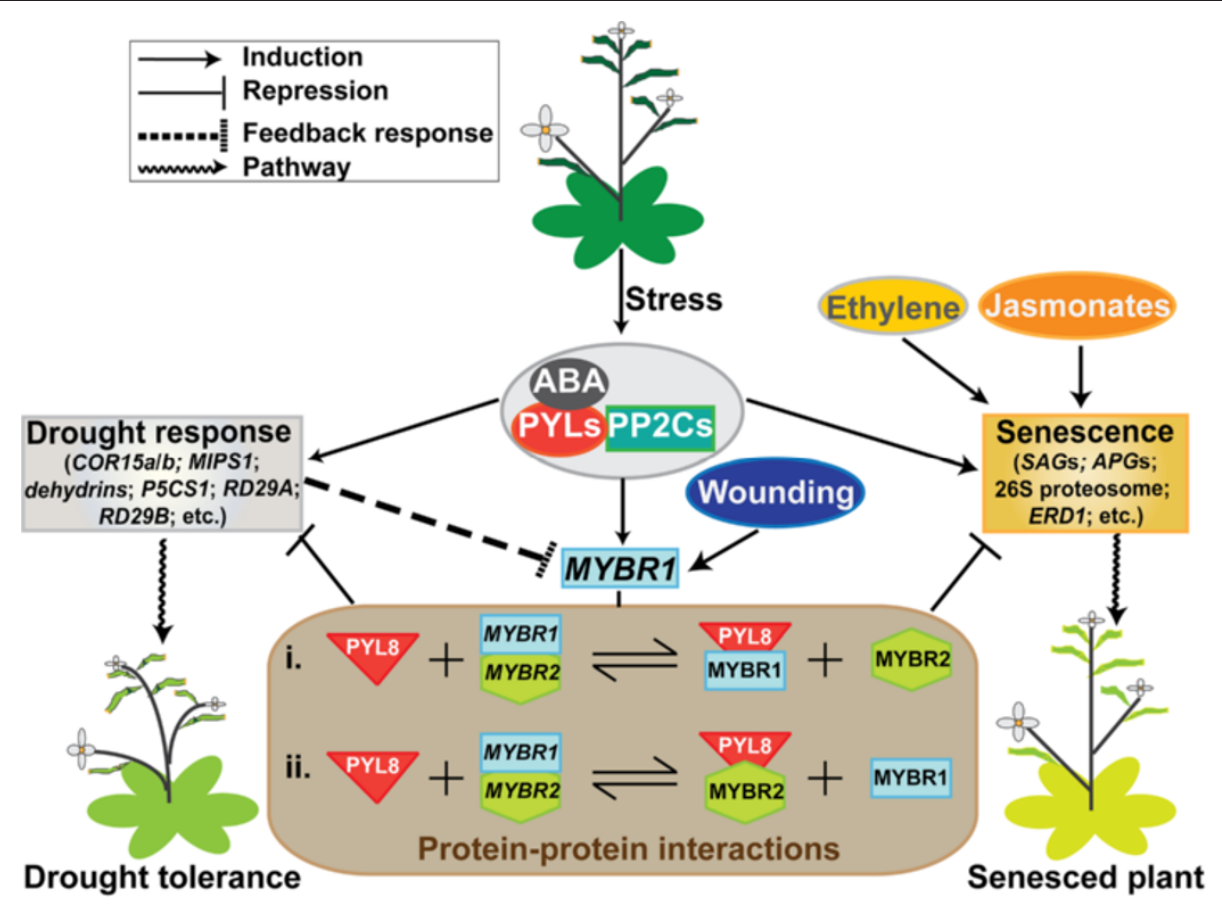

Figure 11 Model of MYBR1 mechanism of action and effects.

biotrophic pathogens and attenuated responses to necrotrophic pathogens.

We propose a model of MYBR1-repression of ABA signaling during drought and senescence (Figure 11). It has been shown previously that PYL8 is localized in both cytoplasm and nucleus and the interaction between PP2C1 and PYL8 takes place in the nucleus [34]. In addition, MYBR1 is also localized in the nucleus [15]. Therefore, the interaction of MYBR1 with PYL8 suggests a direct role of MYBR1 in modulating ABA perception. The uniqueness of the interaction with PYL8 (and with no other PYL) provides an example of receptor specificity - an ABA receptor mediating a specific sub-network of responses. The existence of such effects was suggested by comparison of the effects of ABA analogs in Huang et al. [14]. Previous papers have noted that binding of PYL8 to PP2Cs does not appear to be dependent on $\mathrm{ABA}$, so the regulatory significance of the PYL8-ABA complex is not clear. Increased drought tolerance and $A B A$ hypersensitivity in seed of $35 \mathrm{~S}_{\text {pro }}: P Y L 8$ lines showed that PYL8 is an overall positive regulator of ABA signaling [34]. Binding of MYBR1 to PYL8 may block interaction with and inhibition of PP2Cs. Alternatively, PYL8 may regulate MYBR1 binding to DNA. Since PYL8PP2C binding is independent of ABA, PYL8 may be responsible for constitutive $\mathrm{ABA}$ signaling that is independent of $\mathrm{ABA}$ itself or $\mathrm{ABA}$ may be required to fully potentiate PYL8-PP2C interaction. Future studies will further explore the MYBR1-PYL8 interaction in relation to $M Y B R 1$ function.
The weak phenotypes of the mybr 1 and mybr2 mutants and the enhanced effects in the double mybr $1 \times$ mybr2 mutant strongly suggest that MYBR1 and MYBR2 are partially redundant and the yeast two hybrid data indicates that they may form heterodimers (Figure 11). However, MYBR2 has mainly been associated with auxin signaling and root development [21], shows differing MYBR2 ${ }_{\text {PRO:: }}$ GUS expression patterns compared to MYBR $1_{\mathrm{PRO}}:: \mathrm{GUS}$ [21], and has not been distinctly associated with ABA or jasmonate response as our data and others suggest for MYBR1 [14,15,17,40].The specific interaction of MYBR1 (and not MYBR2) with INO suggests that there are at least some unique functions of MYBR1 not shared by MYBR2. However, the significance of the MYBR1-INO interaction is unknown at this time. INO encodes a YABBY-type transcription factor and is only known to be involved in ovule development [33] and there is no specific MYBR1 phenotype associated with flowers.

The effects of MYBR1 overexpression in Arabidopsis were also studied by Jung et al. [15], but some of their results were significantly different to those reported here. Jung et al. [15] reported downregulation of stress genes but increased stress tolerance and reduced water loss from detached shoots in over-expression lines and obtained similar results in soybean transgenics [25]. Similarly, Persak and Pitzschke [17] reported delayed mortality of an OxMYBR1 line relative to wild type when exposed to toxic levels of salt. For this reason, we focused carefully on identifying the most appropriate approach to measuring 
drought and water loss. We believe that our results demonstrate that the reduced size of OxMYBR1 lines - due to slower growth of above-ground tissues and shorter primary roots - is associated with reduced water use and slower depletion of soil moisture. This phenomenon produced an apparent increase in drought tolerance because the differential size and water use of the MYBR1 genotypes were not taken into account. To circumvent this issue, PEG treatment (which maintains a specific soil water potential) was used to reveal the increased sensitivity of OxMYBR1 lines to water stress (as shown in Figure 3A). Furthermore our microarray results are consistent with reduced stress responses in OxMYBR1 lines and careful analysis of microarray results in Table 1 in Jung et al. [15] suggests that many well-known positive effectors or regulators of stress responses (such as ERD1, KIN1, COR15a, COR15b, RAB18, RESPONSIVE TO DESSICATION29A (RD29A), COR47, RD29B, DELTA1-PYRROLINE-5-CARBOYLATE SYNTHASE1 (P5CS), DREB2A) were similarly downregulated in overexpressing AtMYBR1 plants relative to WT plants. However, Jung et al. did not perform experiments that showed the effects of MYBR1 overexpression on repressing ABA/PBI425-induced genes (as in Figure 1). The differences between our results and Jung et al. [15] in measuring drought tolerance provides a cautionary example of the complexities and subtleties of performing and interpreting drought and water use experiments. Unlike Jung et al. [15] and Persak and Pitzschke [17], we did not investigate salt-stress related phenotypes related to MYBR1 expression. More recently, Jung et al. [40] suggested that MYBR1 was induced non-specifically by phytohormones and suppressed jasmonate responses. Our data also suggest an effect of MYBR1 on repressing JA responses, but show a direct and unambiguous link to ABA signaling as described above.

\section{Conclusions}

In the last few years, considerable information has accumulated on the involvement of MYBR1 in stress-related MAPK signaling. However, the function of the gene in relation to stress responses has remained unclear. This study reveals that MYBR1 is a component of $\mathrm{ABA}$ signaling and appears to be involved in feedback maintenance of adult, pre-senescent growth, especially under conditions of stress and wounding. As such it provides an example of a transcription factor that integrates, balances and co-ordinates hormonal, developmental and environmental signals.

\section{Methods}

Plant materials, growth conditions and treatment

Arabidopsis thaliana plants were grown under long-day conditions in a growth cabinet at $22^{\circ} \mathrm{C}$ and $40 \%$ humidity with $16 \mathrm{~h}$ of $80 \mu \mathrm{E}$ light and $8 \mathrm{~h}$ dark cycles. Seeds were surface sterilized as follows: seeds were washed aseptically; once with 70\% ethanol for $30 \mathrm{sec}$ and three times with $20 \%$ bleach for 5 min followed by four washes with sterile water. Water was removed after the final wash and $0.2 \%$ agar solution was added to facilitate placing seeds on Murashige-Skoog (MS) $+0.8 \%$ agar media without sucrose. Seed stratification was performed at $4{ }^{\circ} \mathrm{C}$, in the dark for $3 \mathrm{~d}$. Since growth rates differ slightly between genotypes, care was taken that observed differences between genotypes at specific times were consistent and not artifacts of different developmental stages.

For microarray experiments, growth of plants, treatment of 5 week old plants with $20 \mu \mathrm{M}$ PBI 425 for $24 \mathrm{~h}$ and above ground tissue collection were done as described in Huang et al. [14].

For root phenotyping of seedlings following seed stratification, agar plates were transferred to a controlled environment cabinet. Eight days after stratification, seedlings were photographed using a digital camera and root lengths were measured using ImageJ software (version $1.37 \mathrm{v}, \mathrm{NIH}, \mathrm{USA})$.

For generation of mybr1xmybr2 double mutant, TDNA insertion lines of (mybr2) SALK_67655 was obtained from the Arabidopsis Stock Center (http://arabidopsis.org). This loss-of-function mutation in this line is caused by TDNA insertion into an exon. mybr2 homozygous plants were identified by PCR as described [42]. Homozygous plants of mybr1 [14] and mybr 2 were crossed reciprocally. Homozygous double mutants mybr1 9 x mybr2 $\hat{\delta}$ and mybr2우 $\mathrm{x} m y b r 1{ }^{\widehat{\jmath}}$ were identified by PCR [42].

\section{PEG treatment}

Following stratification at $4{ }^{\circ} \mathrm{C}$, plants were grown in soil (Sunshine 3 Mix from Sun Gro Horticulture Inc.) for 17 $\mathrm{d}$ in a growth chamber at $22^{\circ} \mathrm{C}$ and $64 \%$ humidity with $16 \mathrm{~h}$ of $150 \mu \mathrm{E}$ light and $8 \mathrm{~h}$ dark cycles, then transplanted individually into 2 "x 2.5 " pots filled with $90 \mathrm{ml}$ sand: soil (2:1) mix. Pots were watered with $30 \mathrm{ml}$ Hoagland solution. We found that maintaining high humidity is crucial in this experiment. Plants were watered as needed and after $20 \mathrm{~d}, 50 \mathrm{ml}$ of $10 \%$ or 15\% PEG solutions was added to each pot. After $30 \mathrm{~min}$ to allow drainage, pots were transferred to fresh tray holders. Pictures were taken $5 \mathrm{~d}$ after PEG treatment.

\section{Transpirational water loss assays of detached whole rosette leaf and whole plants}

Plants were grown as described above. Whole rosette leaves of $20 \mathrm{~d}$ old plants were excised, placed in a weighing boat and weighed at intervals for up to $9 \mathrm{~h}$. Samples were kept at $22^{\circ} \mathrm{C}$ between weighing intervals.

\section{Chlorophyll assay}

Freshly harvested leaves were weighed and chlorophyll was extracted on $0 \mathrm{~d}$ (untreated) and after 6-7 d following dark 
induced senescence. Chlorophyll extraction and quantification were carried out as described by [43]. Leaves or whole rosettes of Arabidopsis were harvested and weighed. Chlorophyll was extracted by placing the tissue in $90 \%$ ethanol at $65^{\circ} \mathrm{C}$ for $3 \mathrm{~h}$ until all tissues became chlorophyll free. The amount of total chlorophyll was determined by measuring absorbance at 664 and $647 \mathrm{~nm}$ [44] with a Microplate Reader (Synergy H1) from Biotek and using the formula: micromoles of chlorophyll per milliliter per gram fresh weight $=7.93\left(\mathrm{~A}_{664}\right)+19.53\left(\mathrm{~A}_{647}\right)$.

\section{MYBR $1_{\text {pro: }}$ GUS plasmid construction, treatments and GUS staining}

A $2.7 \mathrm{~kb}$ fragment, including the 5'UTR, of the AtMYBR1 promoter was PCR amplified from Arabidopsis thaliana (Col-0) WT genomic DNA using the primers 5 '-attB1gtagtgcgtgtggatatatacatgca-3' and 5 '-attB2-tgatttggaatg ttttatcaaactttag-3' and cloned into pDONR221 using a Gateway BP reaction (Invitrogen). Following sequence verification, the MYBR1 promoter was then cloned into the GUS expression vector pMDC162 [45] with an LR reaction (Invitrogen).

For GUS staining in seedling, flower and silique, homozygous $T_{2}$ and $T_{3}$ seedlings were grown for $13 \mathrm{~d}$ on MS medium in the presence of $1 \%$ sucrose and were stained for GUS activity for $70 \mathrm{~min}$. For drought stress, seedlings were grown for 7 days and drought was imposed by overlaying $10 \%$ and $20 \%$ PEG on an agar plate for $44 \mathrm{~h}$ followed by GUS staining for $1 \mathrm{~h}$. True leaves of control plants were wounded aseptically with hemostats and $30 \mathrm{~min}$ GUS staining was performed at $0 \mathrm{~h}$ and after $1 \mathrm{~h}$ of wounding. Floral tissues were stained for $16 \mathrm{~h}$ unless otherwise stated. GUS staining was performed [46] with X-gluc staining reagent ( $0.1 \mathrm{M} \mathrm{NaPO}_{4} \mathrm{pH}_{7.0}, 10 \mathrm{mM}$ $\mathrm{Na}_{2}$ EDTA, $0.1 \%$ Triton X-100, $1.5 \mathrm{mM} \mathrm{K}_{3} \mathrm{Fe}(\mathrm{CN})_{6}$, $0.5 \mathrm{mM} \mathrm{K}_{4} \mathrm{Fe}(\mathrm{CN})_{6}$, and $2.0 \mathrm{mM} \mathrm{X}$-gluc) at $37^{\circ} \mathrm{C}$ in the dark after three vacuum infiltrations of 1 min each. After staining, chlorophyll was removed completely by successive washes with $50 \%, 70 \%$ and $80 \%$ ethanol with gentle agitation and photographs were taken using a Wild M3Z dissecting microscope equipped with a Leica DFC320 camera.

For GUS staining in embryo and endosperm, plants were grown in growth chambers as described above. Siliques were collected at 6, 9, 12, 15 and 18 days post anthesis (DPA) and were fixed in $20 \%$ acetone for $>24 \mathrm{~h}$ at $-20^{\circ} \mathrm{C}$ prior to embryo dissection followed by $30 \mathrm{~min}$ GUS staining. Dry and imbibed seeds at different time points were also fixed, dissected and then stained as described above.

\section{Detached leaf senescence assay}

Plants were grown on soil. Rosette true leaves numbers $1-4$ as counted by order of emergence (cotyledons were excluded), were excised and incubated with their abaxial sides down on two pieces of $3 \mathrm{MM}$ paper wetted with $10 \mathrm{ml}$ of $3 \mathrm{mM}$ MES ( $\mathrm{pH}$ 5.7) without or with different concentration of (+)-ABA, 1-aminocyclopropane-1carboxylic acid (ACC), benzyl amino purine (BP), or MJA at room temperature in the dark [26]. Leaves numbers 1 and 2 were incubated for $5 \mathrm{~d}$ and juvenile leaves numbers 3 and 4 were incubated for 6-13 d. Leaf pictures were taken after treatment and chlorophyll assay (described above) was performed.

\section{Quantification of ABA, cytokinins and their metabolites and JA by LC-MS/MS}

The plant hormone analysis was performed by high performance liquid chromatography-electrospray tandem mass spectrometry (HPLC-ES-MS/MS) using deuterated internal standards, as described $[47,48]$. The analysis of free salicylic and jasmonic acid using HPLC-ES-MS/MS with deuterated internal standards will be presented elsewhere (Han et al., unpublished).

\section{RNA extraction and microarray labeling, hybridization and data acquisition}

Total RNA was extracted from frozen tissues of four independent biological replicates as described [49] with a slight modification. Instead of extraction buffer RLT, a mix containing $10 \mathrm{mM}$ Tris- $\mathrm{HCl} \mathrm{pH} 7.5 ; 0.1 \mathrm{M} \mathrm{NaCl}$; $1 \mathrm{mM}$ EDTA and 1\% SDS was used. RNA quantification was performed by measuring optical density at $260 \mathrm{~nm}$. Microarray labeling, hybridization, scanning and data acquisition were done for oligonucleotide microarrays obtained from the University of Arizona according to Huang et al. [14]. However, microarray labeling, hybridization and slide washing for Agilent Technologies Arabidopsis 4x44k arrays (version 4, product\# G2519F, design ID 021169) were performed according to the manufacturer's protocol using low input Quick Amp Labeling Kit for two color (Agilent Technologies; cat\# 5190-2306) [50]. In short, $200 \mathrm{ng}$ total RNA was used for cDNA synthesis and $2.5 \mathrm{~h}$ for cRNA amplification. Two $\mu$ g each of cyanine 3- and 5labeled amplified cRNA was hybridized to each array. After washing, each slide was scanned using Axon 4000B scanner with a resolution of $5 \mu \mathrm{m} /$ pixel. Data acquisition was done as described above.

\section{Microarray data analysis}

Signal intensity normalization (method: Print-tip loess), filtering bad spots and control spots, filtering minimum channel intensity (intensity for both channel should be $<500$ in most cases) and correlation coefficient among replicates were performed in BASE [51]. Quality control on sample data was performed in GeneSpring GX 10.0.2 (Agilent). To obtain statistically differentially expressed gene sets, a t-test against zero along with Benjamini-Hotchberg multiple 
testing correction and with a 0.05 p-value cut-off were performed in GeneSpring. Furthermore, biologically significant differentially expressed gene sets were obtained by using a threshold fold change $\geq 1.5$. The spot visualization feature in BASE was employed for an additional quality control for false positives/negatives. Afterward, log2 expression values for each sample type were uploaded into MapMan ImageAnnotator version 3.0.0RC3. Analysis for statistically significant enriched biological pathways, a Wilcoxon rank sum t-test embedded in MapMan was performed with a p-value cut-off of 0.05 and Benjamini Hochberg multiple testing correction [52]. Gene annotation was done based on TAIR database, MapMan and PlantsUBQ (URL http://plantsubq.genomics.purdue.edu).

\section{Quantitative RT-PCR}

Gene-specific primers for QRT-PCR were designed using PerlPrimer v1.1.14 [53]; http://perlprimer.sourceforge.net and are listed in Additional file 1: Table S3. Total RNA was isolated as described above, from rosette leaves 3 and 4 of three week old plants. Complementary DNA (cDNA) was produced using $2 \mu \mathrm{g}$ total RNA using QuantiTect Reverse Transcription kit from Qiagen (catalog number 205311) according to the manufacturers instruction. Two biological and two technical repeats were performed with null-template control. Arabidopsis ACTIN2 was used as a normalization control [14]. cDNAs were diluted 10 times in QRT-PCR reactions for all genes (MYBR1, SAG29, SEN1 and SEN4) except SAG12 cDNA which was used without dilution. QRT-PCR was performed with SYBR green SuperScript III Platinum Two-Step qRT-PCR Kit (Invitrogen, 11735-032) according to the manufacturer instructions, on a Stratagene Mx3000P real-time PCR thermal cycler.

\section{Construction of gene fusions for yeast two-hybrid assays} Open reading frames of MYBR1 and MYBR2 and 14 genes of $P Y R / P Y L / R C A R$ s family ABA receptors and the GAL4 activation domain (AD) and DNA-binding domain (BD) were constructed in the pGADT7 and pGBT9 vectors, respectively (Clontech). The open reading frames (ORF) of PYL1/2/3/5/6/7/8/9/10/11/12/13 were PCR amplified from cDNA and the ORF of PYR1 from an ABRC clone (accession number U15941) using PfuUltra II fusion HS DNA polymerase (Agilent; catalog number 600670) and primers are listed in Additional file 1: Table S3. PCR products were gel purified with a gel extraction kit (QIAGEN; catalog number 28704), were cloned into Gateway vector pDONR221 by a Gateway BP reaction (Invitrogen) and were verified by sequencing using M13 forward and reverse primers. ORFs of PYL4 and MYBR2 cloned in pENTR223 were obtained from ABRC clones (accession number G12806 and G14459, respectively) and were verified by sequencing using T7 and M13 forward primers.
These 15 different ORFs were then cloned in-frame with the GAL4AD in pGADT7 by LR reactions (Invitrogen). ORFs of MYBR1 and MYBR2 were cloned in-frame with the GAL4BD in pGBT9 using In-Fusion Advantage PCR Cloning kit (Clontech; catalog number 639619) as follows: MYBR1 ORF was PCR amplified from cDNA and MYBR2 ORF from an ABRC clone G14459 using primers listed in Additional file 1: Table S3. PCR products were gel purified and verified by sequencing using forward $5^{\prime}$ ttttcgttttaaaacctaagagtc- $3^{\prime}$ and reverse $5^{\prime}$-tcatcggaaga gagtagt-3' primers. Plasmid pGBT9 was digested to completion with EcoRI and BamHI and column purified (QIAGEN; catalog number 28106). In-fusion cloning reactions between ORFs and linearized pGBT9 were performed according to the manufacturer's instruction.

\section{Protein-protein interaction analyses}

All gene fusions in pGADT7 and in pGBT9 were transformed into the yeast cell lines Y187 and Y2H Gold, respectively and were grown in the presence of $50 \mu \mathrm{g} / \mu \mathrm{l}$ kanamycin on media SD/Leu and SD/Trp, respectively, according to the manufacturer's instructions (Clontech; Matchmaker gold yeast two-hybrid system; catalog number 630489). Auto-activation and toxicity of pGBT9MYBR1 and pGBT9-MYBR2 were tested as described by Clontech. For library screening, transformed yeast Y2H Gold with pGBT9-MYBR1 was used to screen an Arabidopsis normalized cDNA library; Mate and Plate (Clontech; catalog number 630487) which was constructed from different stages of vegetative and floral tissues, cloned in pGADT7-RecAB vector and transformed into the yeast Y187. After $24 \mathrm{~h}$ mating, library screening was performed on medium SD/-Leu/-Trp/-His/-Ade in the presence of $20 \mu \mathrm{g} / \mathrm{ml} \mathrm{x}$ - $\alpha$-gal (Gold Biotechnology) and $78 \mathrm{ng} / \mathrm{ml}$ Aureobasidin A (Clontech) (QDO/X/A) and grown for $4 \mathrm{~d}$ at $30^{\circ} \mathrm{C}$. Blue yeast colonies were streaked onto fresh QDO/X/A. Following 3 d growth, plasmids were isolated using the Easy Yeast Plasmid Isolation Kit (Clontech) and cDNA inserts were PCR amplified using LD-AD screening primers (Clontech) and verified by sequencing using T7 primer. For individual clone screening, transformed yeast Y2H Gold with pGBT9-MYBR1and pGBT9-MYBR2 and transformed yeast Y187 with each PYR/PYL/RCARs/MYBR2-pGADT7 were mated for $1 \mathrm{~d}$ at $30^{\circ} \mathrm{C}$ and screened on media SD/-Leu/-Trp (DDO), DDO/ $\mathrm{X} / \mathrm{A}$ and $\mathrm{QDO} / \mathrm{X} / \mathrm{A}$ as described by Clontech. Bimolecular fluorescence complementation (BiFC), including preparation of constructs, was performed in $N$. benthamiana epidermal cells according to [50].

\section{Accession numbers}

The Arabidopsis Genome Initiative (AGI) locus identifiers for the genes from this article are as follows: MYBR1/ MYBR44 (At5g67300), MYBR2/MYBR77 (At3g50060), 
PYL8 (At5g53160), INO (At1g23420). SALK T-DNA insertion mutant line of MYBR1 and MYBR2 are SALK 039074 and SALK_67655, respectively.

\section{Additional files}

Additional file 1: Figure S1. Experimental Designs of Two Color Arabidopsis Microarray Experiments using Above-Ground Tissues of 5 Weeks Old Plants. Figure S2. Reduced Water Uptake by Gain of AtMYBR1 Function Leads to Apparent Drought Tolerance in Plants Overexpressing AtMYBR1. Figure S3. Expression of MYBR1 pro:GUS in Vegetative Tissues, Embryos and Endosperm at Different Developmental Stages and after Imbibition of Mature Seeds. Figure S4. Physical Interaction of MYBR1 and MYBR2 with PYL8, MYBR2 and INO in the Presence of Various Hormones and Inhibitors of Auxin Signaling and Transport. Table S2. Enriched TF Sites (http://www.bioinformatics2.wsu.edu/cgi-bin/Athena/cgi/home.pl). Table S3. Primers for QRT-PCR and Gene Cloning for Yeast Two-Hybrid Analysis. Table S5. MYBR1 represses genes associated with leaf senescence. Additional Methods.

Additional file 2: Table S1. Significant Gene List Obtained from T-Test P-Value Cut-Off $\leq 0.05$ and Fold Change $\geq 1.5$ (Excel File).

Additional file 3: Table S4. MYBR1 Represses Genes Induced by Natural Leaf Senescence (Excel File).

\section{Competing interests}

The authors declare that they have no competing interests.

\section{Authors' contributions}

AJC conceived the project. AJC and MRJ designed the experiments and JAF and $\mathrm{DH}$ provided suggestions on experiments. MRJ performed the experiments and analyzed data in the manuscript unless otherwise stated. DH constructed p35Spro:AtMYBR1 plasmid and generated 35Spro:AtMYBR1 $T_{0}$ seeds. JAF constructed PMYBR1pro:GUS plasmid and generated MYBR1pro: GUS To seeds. YL and JAF screened yeast 2-hybrid library. MRJ, YL and JAF verified the interaction of PYL8 with MYBR1. AJC, MRJ, JAF and DH contributed to the interpretation of results. AJC and MRJ wrote and edited the manuscript. All authors read and approved the final manuscript.

\section{Acknowledgements}

Phytohormone analysis was performed by the Plant Hormone Profiling Technology Unit of the Plant Biotechnology Institute - National Research Council of Canada http://www.nrc-cnrc.gc.ca/eng/solutions/advisory/plant_hormone.html by ultra performance liquid chromatography electrospray tandem mass spectrometry (UPLC-ES-MS/MS) employing deuterated internal standards for quantitation. We thank Mr. Chad Matsalla for help with Bioinformatics and Dr. Prakash Venglat for advice in obtaining the images in Figure 11. We thank Dr. Suzanne Abrams, for providing (+)-ABA and ABA analog (+)-8' acetylene ABA (PBI425). We thank Dr. Qing Lu and Dr. Yuhai Cui (AAFC in London, Ontario) for providing a Gateway-compatible version of vector pGADT7. We are grateful to Dr. Raju Datla and Dr. Ed Tsang for helpful comments during the preparation of the manuscript. Funding for some of this research was provided by Genome Canada and Genome Prairie as part of the project 'enhancing canola through genomics'. This paper is NRCC number 50185

\section{Author details}

${ }^{1}$ Plant Biotechnology Institute, National Research Council of Canada, 110 Gymnasium Place, Saskatoon S7N 0W9, Canada. ${ }^{2}$ Zhejiang Agriculture and Forestry University, Hangzhou 311300, China.

Received: 9 September 2013 Accepted: 21 November 2013 Published: 28 November 2013

\section{References}

1. Cutler SR, Rodriguez PL, Finkelstein RR, Abrams SR: Abscisic acid: Emergence of a core signaling network. Annu Rev Plant Biol 2010, 61:651-679.
2. Ma Y, Szostkiewicz I, Korte A, Moes D, Yang Y, Christmann A, Grill E: Regulators of PP2C phosphatase activity function as abscisic acid sensors. Science 2009, 324:1064-1068

3. Park SY, Fung P, Nishimura N, Jensen DR, Fujii H, Zhao Y, Lumba S, Santiago J, Rodrigues A, Chow TF, et al: Abscisic acid inhibits type $2 \mathrm{C}$ protein phosphatases via the PYR/PYL family of START proteins. Science 2009, 324:1068-1071.

4. Santiago J, Rodrigues A, Saez A, Rubio S, Antoni R, Dupeux F, Park SY, Márquez JA, Cutler SR, Rodriguez PL: Modulation of drought resistance by the abscisic acid receptor PYL5 through inhibition of clade A PP2Cs. Plant J 2009, 60:575-588

5. Yin P, Fan H, Hao Q, Yuan X, Wu D, Pang Y, Yan C, Li W, Wang J, Yan N: Structural insights into the mechanism of abscisic acid signaling by PYL proteins. Nat Struct Mol Biol 2009, 16:1230-1236.

6. Bartels D, Sunkar R: Drought and salt tolerance in plants. Crit Rev Plant SCi 2005, 24:23-58.

7. Yamaguchi-Shinozaki K, Shinozaki K: Transcriptional regulatory networks in cellular responses and tolerance to dehydration and cold stresses. Annu Rev Plant Biol 2006, 57:781-803.

8. Huang D, Wu W, Abrams SR, Cutler AJ: The relationship of drought-related gene expression in Arabidopsis thaliana to hormonal and environmental factors. J Exp Bot 2008, 59:2991-3007.

9. Lim PO, Kim HJ, Nam HG: Leaf senescence. Annu Rev Plant Biol 2007, 58:115-136

10. Rivero RM, Kojima M, Gepstein A, Sakakibara H, Mittler R, Gepstein S, Blumwald E: Delayed leaf senescence induces extreme drought tolerance in a flowering plant. Proc Natl Acad Sci USA 2007, 104:19631-19636.

11. Munné-Bosch S, Alegre L: Die and let live: leaf senescence contributes to plant survival under drought stress. Funct Plant Biol 2004, 31:203-216.

12. Riechmann JL, Ratcliffe OJ: A genomic perspective on plant transcription factors. Curr Opin Plant Biol 2000, 3:423-434.

13. Stracke $R$, Werber $M$, Weisshaar B: The R2R3-MYB gene family in Arabidopsis thaliana. Curr Opin Plant Biol 2001, 4:447-456.

14. Huang D, Jaradat MR, Wu W, Ambrose SJ, Ross AR, Abrams SR, Cutler AJ: Structural analogs of $A B A$ reveal novel features of $A B A$ perception and signaling in Arabidopsis. Plant J 2007, 50:414-428.

15. Jung C, Seo JS, Han SW, Koo YJ, Kim CH, Song SI, Nahm BH, Choi YD, Cheong JJ: Overexpression of AtMYB44 enhances stomatal closure to confer abiotic stress tolerance in transgenic Arabidopsis. Plant Physiol 2008, 146:623-635.

16. Pitzschke A, Djamei A, Teige M, Hirt H: VIP1 response elements mediate mitogen-activated protein kinase 3-induced stress gene expression. Proc Natl Acad Sci 2009, 106:18414-18419.

17. Persak H, Pitzschke A: Tight Interconnection and Multi-Level Control of Arabidopsis MYB44 in MAPK Cascade Signalling. PLoS ONE 2013, 8:e57547.

18. Nguyen XC, Hoang MHT, Kim HS, Lee K, Liu XM, Kim SH, Bahk S, Park HC, Chung WS: Phosphorylation of the transcriptional regulator MYB44 by mitogen activated protein kinase regulates Arabidopsis seed germination. Biochem and Biophys Res Commun 2012, 423:703-708.

19. Cutler AJ, Rose PA, Squires TM, Loewen MK, Shaw AC, Quail JW, Krochko JE, Abrams SR: Inhibitors of abscisic acid 8'-hydroxylase. Biochemistry 2000 39:13614-13624.

20. Kirik V, Kolle K, Miséra S, Bäumlein H: Two novel MYB homologues with changed expression in late embryogenesis-defective Arabidopsis mutants. Plant Mol Biol 1998, 37:819-827.

21. Shin R, Burch AY, Huppert KA, Tiwari SB, Murphy AS, Guilfoyle TJ, Schachtman DP: The Arabidopsis transcription factor MYB77 modulates auxin signal transduction. Plant Cell 2007, 19:2440-2453.

22. van der Graaff E, Schwacke R, Schneider A, Desimone M, Flügge UI, Kunze R: Transcription analysis of Arabidopsis membrane transporters and hormone pathways during developmental and induced leaf senescence. Plant Physiol 2006, 141:776-792.

23. Yamaguchi-Shinozaki K, Shinozaki K: Organization of cis-acting regulatory elements in osmotic- and cold-stress-responsive promoters. Trends Plant Sci 2005, 10:88-94.

24. O'Connor TR, Dyreson C, Wyrick JJ: Athena: a resource for rapid visualization and systematic analysis of Arabidopsis promoter sequences. Bioinformatics 2005, 21:4411-4413.

25. Seo J, Sohn H, Noh K, Jung C, An J, Donovan C, Somers D, Kim D, Jeong SC, Kim CG, et al: Expression of the Arabidopsis AtMYB44 gene confers drought/ salt-stress tolerance in transgenic soybean. Mol Breeding 2012, 29:601-608. 
26. Doelling $\mathrm{JH}$, Walker JM, Friedman EM, Thompson AR, Vierstra RD: The APG8/12-activating enzyme APG7 is required for proper nutrient recycling and senescence in Arabidopsis thaliana. J Biol Chem 2002, 277:33105-33114

27. Buchanan-Wollaston V, Page T, Harrison E, Breeze E, Lim PO, Nam HG, Lin JF, Wu SH, Swidzinski J, Ishizaki K, et al: Comparative transcriptome analysis reveals significant differences in gene expression and signalling pathways between developmental and dark/starvation-induced senescence in Arabidopsis. Plant J 2005, 42:567-585.

28. Kim HS, Delaney TP: Arabidopsis SON1 is an F-Box protein that regulates a novel induced defense response independent of both salicylic acid and systemic acquired resistance. Plant Cell 2002, 14:1469-1482.

29. Sakakibara H: Cytokinins: activity, biosynthesis, and translocation. Annu Rev Plant Biol 2006, 57:431-449.

30. Hwang I, Sheen J: Two-component circuitry in Arabidopsis cytokinin signal transduction. Nature 2001, 413:383-389.

31. Chaudhury AM, Letham S, Craig S, Dennis ES: amp1 - a mutant with high cytokinin levels and altered embryonic pattern, faster vegetative growth, constitutive photomorphogenesis and precocious flowering. Plant J 1993, 4:907-916.

32. Werner T, Motyka V, Strnad M, Schmülling T: Regulation of plant growth by cytokinin. Proc Natl Acad Sci USA 2001, 98:10487-10492.

33. Villanueva JM, Broadhvest J, Hauser BA, Meister RJ, Schneitz K, Gasser CS: INNER NO OUTER regulates abaxial-adaxial patterning in Arabidopsis ovules. Genes Dev 1999, 13:3160-3169.

34. Saavedra X, Modrego A, Rodríguez D, González-García MP, Sanz L, Nicolás G, Lorenzo O: The nuclear interactor PYL8/RCAR3 of Fagus sylvatica FsPP2C1 is a positive regulator of abscisic acid signaling in seeds and stress. Plant Physiol 2010, 152:133-150.

35. Pandey GK, Grant JJ, Cheong YH, Kim BG, Li L, Luan S: ABR1, an APETALA2domain transcription factor that functions as a repressor of $A B A$ response in Arabidopsis. Plant Physiol 2005, 139:1185-1193.

36. Song CP, Agarwal M, Ohta M, Guo Y, Halfter U, Wang P, Zhu JK: Role of an Arabidopsis AP2/EREBP-type transcriptional repressor in abscisic acid and drought stress responses. Plant Cell 2005, 17:2384-2396.

37. Himmelbach A, Hoffmann $T$, Leube $M$, Höhener B, Grill E: Homeodomain protein ATHB6 is a target of the protein phosphatase $A B I 1$ and regulates hormone responses in Arabidopsis. EMBO J 2002, 21:3029-3038.

38. Allègre $M$, Daire $X$, Héloir MC, Trouvelot $S$, Mercier L, Adrian M, Pugin A: Stomatal deregulation in Plasmopara viticola-infected grapevine leaves. New Phytol 2007, 173:832-840.

39. Gan S, Amasino RM: Inhibition of leaf senescence by autoregulated production of cytokinin. Science 1995, 270:1986-1988.

40. Jung C, Shim J, Seo J, Lee H, Kim C, Choi Y, Cheong JJ: Non-specific phytohormonal induction of AtMYB44 and suppression of jasmonateresponsive gene activation in Arabidopsis thaliana. Mol Cells 2010, 29:71-76.

41. Shim JS, Jung C, Lee S, Min K, Lee YW, Choi Y, Lee JS, Song JT, Kim JK, Choi YD: AtMYB44 regulates WRK70 expression and modulates antagonistic interaction between salicylic acid and jasmonic acid signaling. Plant J 2013, 73:483-495.

42. Alonso JM, Stepanova AN, Leisse TJ, et al: Genome-wide insertional mutagenesis of Arabidopsis thaliana. Science 2003, 301:653-657.

43. Lolle SJ, Berlyn GP, Engstrom EM, Krolikowski KA, Reiter WD, Pruitt RE: Developmental regulation of cell interactions in the Arabidopsis fiddlehead-1 mutant: a role for the epidermal cell wall and cuticle. Dev Biol 1997, 189:311-321.

44. Hiscox JD, Israelstam GF: A method for the extraction of chlorophyll from leaf tissue without maceration. Can J Bot 1979, 57:1332-1334.

45. Curtis MD, Grossniklaus U: A gateway cloning vector set for high-throughput functional analysis of genes in planta. Plant Physiol 2003, 133:462-469.

46. Jefferson RA, Kavanagh TA, Bevan MW: GUS fusions: $\beta$-glucuronidase as a sensitive and versatile gene fusion marker in higher plants. EMBO J 1987, 6:3901-3907.

47. Chiwocha SDS, Abrams SR, Ambrose SJ, Cutler AJ, Loewen M, Ross ARS, Kermode AR: A method for profiling classes of plant hormones and their metabolites using liquid chromatography-electrospray ionization tandem mass spectrometry: an analysis of hormone regulation of thermodormancy of lettuce (Lactuca sativa L.) seeds. Plant J 2003, 35:405-417.

48. Kong L, Abrams SR, Owen SJ, Graham H, Von Aderkas P: Phytohormones and their metabolites during long shoot development in Douglas-fir following cone induction by gibberellin injection. Tree Physiol 2008, 28:1357-1364

49. Fei $H$, Tsang E, Cutler AJ: Gene expression during seed maturation in Brassica napus in relation to the induction of secondary dormancy. Genomics 2007, 89:419-428.

50. Feurtado JA, Huang D, Wicki-Stordeur L, Hemstock LE, Potentier MS, Tsang EWT, Cutler AJ: The Arabidopsis C2H2 Zinc Finger INDETERMINATE DOMAIN1/ ENHYDROUS Promotes the Transition to Germination by Regulating Light and Hormonal Signaling during Seed Maturation. Plant Cell 2011, 23:1772-1794.

51. Saal LH, Troein C, Vallon-Christersson J, Gruvberger S, Borg A, Peterson C BioArray Software Environment (BASE): a platform for comprehensive management and analysis of microarray data. Genome Biol 2002, 3:software0003.1-software0003.6.

52. Thimm $O$, Bläsing $O$, Gibon $Y$, Nagel A, Meyer S, Krüger $P$, Selbig J, Müller LA Rhee SY, Stitt M: MAPMAN: a user-driven tool to display genomics data sets onto diagrams of metabolic pathways and other biological processes. Plant J 2004, 37:914-939.

53. Marshall OJ: PerlPrimer: cross-platform, graphical primer design for standard, bisulphite and real-time PCR. Bioinformatics 2004, 20:2471-2472.

doi:10.1186/1471-2229-13-192

Cite this article as: Jaradat et al:: Multiple roles of the transcription factor AtMYBR1/AtMYB44 in ABA signaling, stress responses, and leaf senescence. BMC Plant Biology 2013 13:192

\section{Submit your next manuscript to BioMed Central and take full advantage of:}

- Convenient online submission

- Thorough peer review

- No space constraints or color figure charges

- Immediate publication on acceptance

- Inclusion in PubMed, CAS, Scopus and Google Scholar

- Research which is freely available for redistribution 